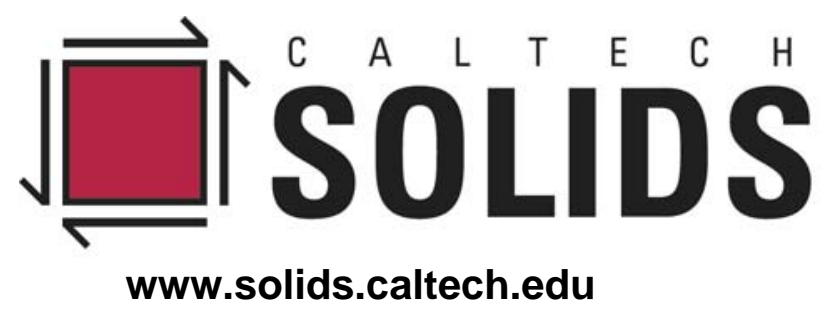

\title{
A COARSE-GRAINED MODEL OF THE MYOFIBRIL: OVERALL DYNAMICS AND THE EVOLUTION OF SARCOMERE NON-UNIFORMITIES
}

S. Givli, and K. Bhattacharya

November 4, 2008 


\title{
A Coarse-Grained Model of the Myofibril: Overall Dynamics and the Evolution of Sarcomere Non-Uniformities
}

\author{
Sefi Givli ${ }^{* \dagger}$ and Kaushik Bhattacharya ${ }^{\S}$ \\ ${ }^{*}$ Faculty of Mechanical Engineering, Technion - Israel Institute of Technology, Haifa 32000, Israel \\ ${ }^{\dagger}$ Corresponding Author, Fax: +972 (4) 829-5711. Email: mesefi@tx.technion.ac.il \\ $\S$ Division of Engineering and Applied Science, California Institute of Technology, CA 91125, USA
}

\begin{abstract}
$\underline{\text { Abstract }}$
A theoretical framework for predicting the macroscopic behavior of a muscle myofibril based on the collective behavior of sarcomeres is presented. The analysis is accomplished by rigorously transforming the nonlinear dynamics of an assemblage of sarcomeres into a partial differential equation for the probability distribution function of sarcomere lengths in the presence of stochastic temporal fluctuations and biological variability. This enables the study of biologically relevant specimens with reasonable computational effort. The model is validated by a comparison to quantitative experimental results. Further, it reproduces experimental observations that can not be explained by standard single sarcomere models, and provides new insights into muscle function and muscle damage during cyclic loading. We show that the accumulation of overstretched sarcomeres, which is related to muscle damage, depends on a delicate interplay between the dynamics of a large number of sarcomeres and the load characteristics, such as its magnitude and frequency. Further, we show that biological variability rather than stochastic fluctuations are the main source for sarcomere non-uniformities.
\end{abstract}

Keywords: Muscle; Sarcomere length; Fokker-Planck ; Distribution; instability, probability.

\section{Introduction}

The muscle is a hierarchical structure with structural components that span over several scales. The basic contractile unit of the muscle is the sarcomere that has a length of two microns. The sarcomere is made of multiple actin and myosin filaments, and myosin heads "pull" on the actin filaments to produce contraction. Myofibrils, typically several millimeters long and less than a micron thick, are composed of thousands of sarcomeres connected in series. In turn, the single muscle cell (or muscle fiber) contains a large number of aligned myofibrils. Finally, the whole muscle is made of bundles of muscle fibers coupled by tissue.

The mechanical response of single muscle fibers (and of whole muscles) has been exhaustively investigated in the past century by various experimental methods. Representative examples are the works of Edman (1999), Edman et al. (1997), Gordon et al. (1966a; b), Hill (1938), Katz (1939), Lombardi and Piazzesi (1990, 1992), and Piazzesi et al. (1992) in which the isometric tension and stiffness were obtained as a function of length and the force-velocity 
relation was measured. Further, the structure of the single sarcomere has been established down to the molecular level especially in recent decades (e.g. Dobbie, et al., 1998; Dunaway, et al., 2002; Huxley and Niedergerke, 1954; Huxley and Hanson, 1954; Irving, et al., 1992; Irving, et al., 2000; Piazzesi, et al., 2002; Wray, 1979). These observations have led to theoretical models of the cross-bridge cycle and of the single sarcomere dynamics (Daniel, et al., 1998; Huxley, 1957; Lombardi and Piazzesi, 1990; Marland, 1998; Pate and Cooke, 1989; Thorson and White, 1969; White and Thorson, 1973).

Consequently, the mechanics of the muscle at the macro (muscle-fiber) and at the micro (single sarcomere) levels is well-understood. Nevertheless, there is a lack of a theory that links the different scales. The need for such a theory is emphasized by the fact that single-sarcomere theories can not explain some experimental observations on the macro-scale (Edman and Reggiani, 1984; Gordon, et al., 1966a; b; Harry, et al., 1990; Julian and Morgan, 1979a; b; Morgan and Proske, 1984; Mutungi and Ranatunga, 2000). These include creep - gradual increase in tension under isometric conditions, permanent extra tension - a higher tension level maintained after a rapid stretch followed by shortening to the original fiber length, and length redistribution - some fiber segments lengthen at the expense of other segments in the same fiber. It has long been speculated that these phenomena are dominated by the non-uniformities in sarcomere length.

There is much experimental evidence for the existence of non-uniformities in sarcomere lengths along the myofibril (e.g. Edman and Reggiani, 1984; Gordon, et al., 1966a; Julian and Morgan, 1979a; b; Lombardi and Piazzesi, 1992; Piazzesi, et al., 1992). These non-uniformities stem from two coupled sources:

1. The sarcomere is bistable under quasistatic conditions (Gordon, et al., 1966a; b; Harry, et al., 1990; Julian and Morgan, 1979a; b; Marland, 1998; Morgan and Proske, 1984; Piazzesi, et al., 1992). The bistability resembles the double well energy structure of a phase transforming solid. It is therefore expected that the sarcomeres could evolve into two groups (or phases) under appropriate loading conditions.

2. The force capacity of the sarcomeres can vary due to two possible mechanisms: (a) Slow time effects, attributed mainly to non-homogeneities in structural properties due to biological variability. Typical examples are the variation in the myofibril diameter, and the dispersion in passive stiffness. (b) Fast time effects that lead to rapid fluctuations in the force capacity 
of the sarcomere. Examples are the rapid fluctuations in Calcium ions concentration, and the rapid attachments and detachments of cross-bridges.

It is important to note that these two factors are coupled: even small variations in the force capacity can result in profound effects on the overall behavior of the myofibril due to the bistability of the sarcomeres.

In reality, muscles operate far from quasistatic conditions, and dynamic instability rather than static instability dominate the response (Denoth, et al., 2002; Zahalak, 1997). In the past three decades there has been an effort to quantitatively explain the unusual phenomena like creep and length-redistribution observed in macro-scale experiments by modeling the myofibril as a system of sarcomeres that are connected in series (Allinger, et al., 1996; Denoth, et al., 2002; Edman and Reggiani, 1984; Marland, 1998; Morgan, 1990; Morgan, et al., 1982). All these models consider the myofibril as a discrete system of sarcomeres, and track (or solve for) each and every sarcomere. This becomes a complex task and is numerically expensive for any physiologically relevant number of sarcomeres. Indeed, some of the studies mentioned above limit their analysis to less than 12 sarcomeres, which is far from representative. Thus, a conclusive analysis of the issues raised above remains lacking.

In the current paper, we take advantage of the fact that the myofibril comprises a large ensemble of sarcomeres. Specifically, we study the evolution of the distribution of the sarcomere lengths instead of tracking each sarcomere. This is made possible by the evidently simple observation that the sarcomeres are connected in series in a myofibril, and thus the behavior of the ensemble may be obtained by constant force averaging. We show that if the sarcomeres are uniform and not subjected to fluctuations, the length distribution function is governed by a simple conservative advection law. Further, if the sarcomeres are physiologically non-uniform or subjected to fluctuations, then the length distribution follows a nonlinear conservation law or a Fokker-Planck equation, respectively. Solving the partial differential equation that governs the sarcomere length distribution leads to the overall response of the myofibril, but also provides important information on inter-sarcomere dynamics, which can be used to explain and also quantify interesting experimental observations such as creep, sarcomere length redistribution, sarcomere popping and more. We note that our approach has several advantages. It enables us to focus directly on statistical quantities and overall response. This significantly reduces computation effort, but importantly provides insight and intuition. 
Clearly, the quality of the overall model depends on that of the micro-level (sarcomere) model. The approach we follow is applicable to a very general class of sarcomere models. However, for specifity, we use the model of Denoth et al. (2002) for elaboration. This model includes three main components: an active component representing the cross-bridges interactions, a passive component representing the passive elements in the sarcomere, such as the titin molecule, and an elastic component representing the elasticity of the filaments. We also use the mechanical properties assigned by Denoth et al. (2002) based on published experimental observations.

The paper is organized in the following order: Section 2 discusses the single sarcomere model. Section 3 presents the model of a myofibril which is an ensemble of sarcomeres connected in series. We derive a partial differential equation for the length distribution when the sarcomeres are uniform (Model H, Section 3.3.1), when sarcomeres suffer from stochastic fluctuations (Model F, Section 3.3.2), and when sarcomeres are physiologically non-uniform (Model V, Section 3.3.3). Sections 4 and 5 present the numerical procedure and selected results, respectively. The numerical examples are chosen to validate the model against published experimental results, and in order to demonstrate unusual phenomena, such as creep, sarcomere popping, homogenization, and length redistribution. In addition, the numerical simulations are oriented towards possible design of new experiments, and analysis of muscle damage associated with eccentric contraction (active lengthening of the muscle). It is widely recognized that eccentric contraction is the cause of muscle damage with the symptom of delayed onset muscle soreness (Morgan and Allen, 1999; Morgan and Proske, 2004), and that the level of damage is directly related to the number of overstretched sarcomeres with no overlap between the thin and thick filaments. Therefore, a key parameter included in the numerical results is the fraction of overstretched sarcomeres. In Section 6, a brief summary is provided and the main conclusions are discussed. 


\section{A single sarcomere}

\subsection{Schematic description}

The sarcomere is the basic contractile unit of muscles. It is typically 2 microns in length and 1 micron in diameter. The sarcomere is mainly made of thin filaments (actin), thick filaments (myosin) and titin. In skeletal muscles, the thin and thick filaments are arranged in hexagonal arrays through the cross-section of the sarcomere and form a "striation pattern" in the longitudinal direction. Sarcomere contraction occurs due to sliding of the two types of filaments relative to each other. Force is generated by myosin heads that form cross-bridges by attaching to specific binding sites on the thin filaments, as illustrated schematically in Fig. 1. In a schematic description, the myosin heads act as hands pulling on a rope (the thin filament) as follows: (i) the myosin head attaches to an actin binding site, (ii) the head generates a "pulling force" by changing its conformation, and (iii) the myosin head detaches from the thin filament. During this "cross-bridge cycle" the myosin head goes through several chemical states, changes conformation, and hydrolyses ATP. Details of this cycle can be found in many textbooks and papers, (e.g., Irving, et al., 2000; Keener and Sneyd, 1998; Lombardi and Piazzesi, 1990; Pate and Cooke, 1989). In addition to the thin and thick filaments the sarcomere includes titin - a long molecule that spans the length of the sarcomere. The main purpose of the titin is to provide additional structural stability to the sarcomere.

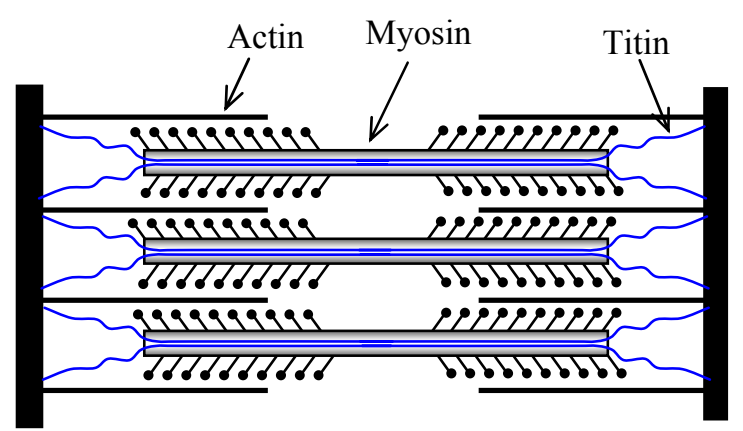

Relaxed

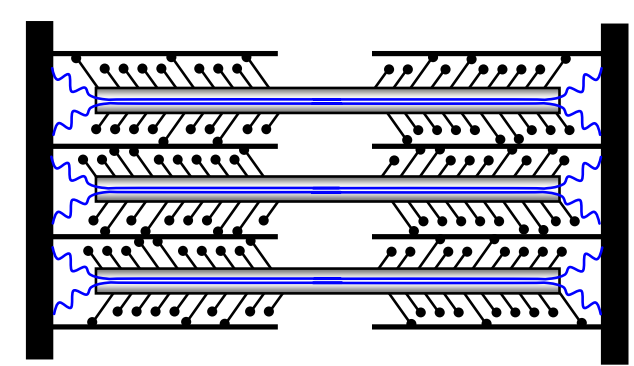

Contracted

Fig. 1: Schematic illustration of a single sarcomere. Contraction occurs due to relative sliding between the thin (actin) and thick (myosin) filaments, and force is generated by myosin heads that bind to the thin filaments and form cross-bridges. 


\subsection{A specific model of the single sarcomere}

Various models of a single sarcomere have been discussed in the literature. While our approach is broadly applicable, we consider for specificity the phenomenological model described in Appendix A.3. This model is similar to the one proposed by (Denoth, et al., 2002) but incorporates two simplifications: (i) the thin and thick filaments are considered inextensible. (ii) the force-velocity relation of the passive element is assumed to be similar to that of the active element. The accuracy of these simplifications is discussed in Appendix A. According to this model, the total contractile force generated by the sarcomere is given as

$$
F=\left(F_{A 1}(L)+F_{T 1}(L)\right) \cdot \phi(\dot{L})=F_{1}(L) \cdot \phi(\dot{L}),
$$

where $\mathrm{L}$ is the sarcomere length. The function $\mathrm{F}_{1}(\mathrm{~L})$ describes the contractile force generated by the sarcomere when it is held at a fixed length and the muscle is fully activated (isometric conditions). This isometric force, $\mathrm{F}_{1}$, is the sum of an active force, $\mathrm{F}_{\mathrm{A} 1}$, generated by the crossbridges and of a passive force, $\mathrm{F}_{\mathrm{T} 1}$, attributed mainly to titin, at a fixed sarcomere length, $\mathrm{L}$. The non-dimensional function $\phi(\dot{L})$ is an effective velocity corrector. The explicit forms of these functions are provided in Appendix A and illustrated in Fig. 2 and Fig. 3. All forces throughout the paper are normalized by the maximal active isometric force, $\mathrm{F}_{0}$.

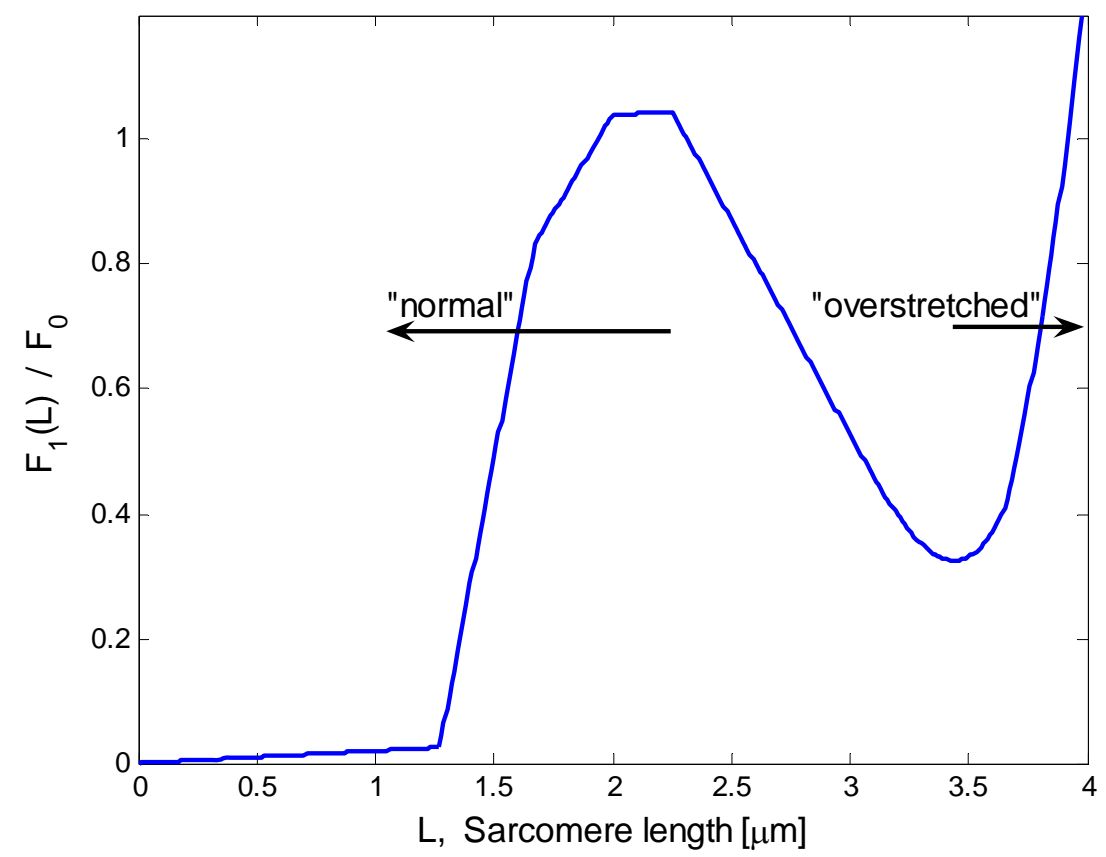

Fig. 2: Force-length relation of a single sarcomere under isometric conditions. 
Recall that the force is generated by the myosin heads pulling on the actin filament through the cross-bridge cycle. The function $\phi$ is an effective description of the rate of the myosin crossbridge cycle. The function $\mathrm{F}_{1}$ describes the overlap between the thin and thick filaments and thus represents the number of participating myosin heads. This motivates the assumed multiplicative decomposition (1). We note that at lengths larger than $3.5 \mu \mathrm{m}$ there is no overlap between the thin and thick filaments, and unfolding and stretch dynamics of titin dominate the sarcomere behavior. This might lead to some deviation from the multiplicative decomposition (1) for these lengths.

The force-length relation is divided into four regions: (1) The ascending-limb for lengths smaller than $2 \mu \mathrm{m}$. (2) The plateau, at lengths between 2 and $2.25 \mu \mathrm{m}$. (3) The descending-limb, at lengths between 2.25 and $3.5 \mu \mathrm{m}$. (4) The overstretched-limb for lengths above $3.5 \mu \mathrm{m}$. The descending limb differs from the other regions by being statically unstable. The stable states correspond to normal operating lengths where a significant overlap exists between the thin and thick filaments, and overstretched sarcomere lengths where there is no overlap between the filaments and force is generated only by the passive elements.

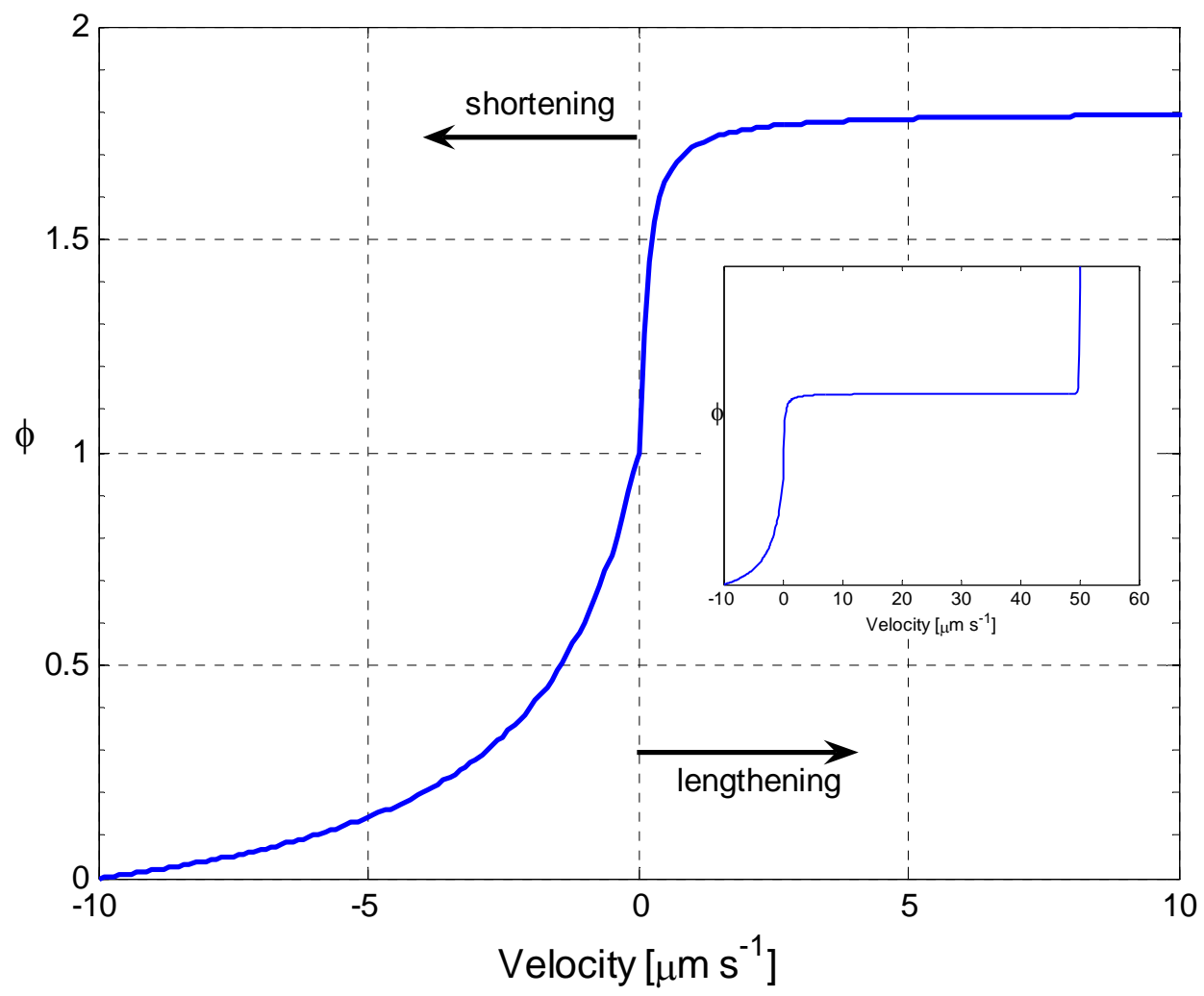

Fig. 3: The force-velocity relation of a single sarcomere at ideal length. The force is normalized by the isometric force. Negative velocities correspond to shortening. 
The main characteristics of $\phi$ are that it is strictly monotonously increasing, the value of $\phi$ at $\dot{L}=0$ is one (under isometric conditions the contractile force equals $F_{1}(L)$ ), the derivative of $\phi$ at $\dot{L}=0$ is discontinuous with a higher value on the right side, at moderate to high velocities the force generated by the sarcomere is approximately constant with a value approaching $1.8 \mathrm{~F}_{0}$, and $\phi$ increases steeply with increasing velocity at high velocities, due to viscous effects (inset of Fig. $3)$.

The function $\phi$ is invertible due to its monotonicity. Therefore, we can rewrite relation (1) in the form

$$
\dot{L}=v\left(\frac{F(t)}{F_{1}(L)}\right)
$$

where $\mathrm{v}$ is the inverse function of $\phi$ i.e., $v(\phi(x))=x$

\subsection{General models}

There are other more general models of sarcomeres in the literature. We show in Appendix A.2 that all of them can be written in the form

$$
\dot{L}=v(L, F(t)) .
$$

Here, $L$ is possibly a vector valued length that represents the sarcomere state parameters (e.g. sarcomere length and overlap length between thin and thick filaments). We observe that our coarse-grained approach only uses this form, equation (3), and is thus applicable to all general models.

\section{Coarse-grained model of a myofibril}

The sarcomeres are connected in a series to form a myofibril, and inertial effects are negligible (Denoth, et al., 2002). It follows that all sarcomeres in a myofibril experience the same force

$$
F^{(i)}(t)=F_{\text {ext }}(t) \quad \forall i, t
$$

where $\mathrm{F}^{(\mathrm{i})}$ is the contractile force generated by the $\mathrm{i}$-th sarcomere, see equation (1), and $\mathrm{F}_{\text {ext }}$ is the external force exerted on the myofibril. Experimental protocols of muscle fibers involve either 
force control or length control conditions. The first, where an external force is applied and the overall length is measured, is easy to simulate (since $F(t)$ is explicitly known) but difficult to perform. The second, where the length is controlled and the force measured, is easier to perform and more common. It introduces the constraint

$$
L_{\text {myofibril }}=\sum L^{(i)}
$$

which is used to compute $\mathrm{F}_{\text {ext }}(\mathrm{t})$.

In the next three sections we present three different models for describing the collective behavior of the sarcomeres in a myofibril under three physical settings.

\subsection{Model $\mathrm{H}$ - homogeneous ensemble}

This model assumes that all sarcomeres along the myofibril have identical mechanical properties, and that the sarcomeres do not undergo fluctuations in their force capacity. Yet, the model assumes that the sarcomeres have some initial length non-uniformities. We note that this model is somewhat naïve as it does not address the sources for sarcomere length non-uniformities. Nevertheless, this model is useful as a model of reference. In this model, the dynamics of each sarcomere is governed by (2). Together with (4), the behavior of the i-th sarcomere in a myofibril is given as

$$
\dot{L}^{(i)}=v\left(\frac{F_{\text {ext }}(t)}{F_{1}\left(L^{(i)}\right)}\right)
$$

We define the sarcomere length probability density function or length distribution, $\mathrm{P}(\mathrm{L}, \mathrm{t})$, as the density of the sarcomeres at length $\mathrm{L}$ and time $\mathrm{t}$. Hence, the fraction of sarcomeres at time $\mathrm{t}$ which have a length in the interval $\left[\mathrm{L}_{1}, \mathrm{~L}_{2}\right]$ is

$$
\text { fraction in }\left[L_{1}, L_{2}\right] \text { at time } t=\int_{L_{1}}^{L_{2}} P(L, t) d L \text {. }
$$

The fraction of sarcomeres in this interval can change only because of addition or removal of sarcomeres across the end-points of the interval. The rate of flow or flux of sarcomeres at a length $\mathrm{L}$ at time $\mathrm{t}$ is 


$$
J(L, t)=P(L, t) V(L, t) \quad \text { with } \quad V(L, t)=v\left(\frac{F_{\text {ext }}(t)}{F_{1}(L)}\right)
$$

Hence, the rate of change in the fraction of the sarcomeres with lengths in the interval $\left[\mathrm{L}_{1}, \mathrm{~L}_{2}\right]$ is given by

$$
\frac{d}{d t}\left(\int_{L_{1}}^{L_{2}} P(L, t) d L\right)=P\left(L_{1}, t\right) V\left(L_{1}, t\right)-P\left(L_{2}, t\right) V\left(L_{2}, t\right) .
$$

Equation (9) constitutes an integral form of a conservation law for P. Assuming that P and V are differentiable functions and using the fact that the interval $\left[\mathrm{L}_{1}, \mathrm{~L}_{2}\right]$ is arbitrary, the differential form of the conservation law is obtained to be:

$$
\frac{\partial P(L, t)}{d t}+\frac{\partial}{d L}(P(L, t) V(L, t))=0
$$

Equation (10) has the form of the conservative advection equation (LeVeque, 1998; 2002), which governs the dynamics of various physical problems. Simple examples are the advection of an incompressible fluid in a pipe, and the flow of traffic. Therefore, the function $\mathrm{V}$ has an effect similar to that of a velocity field acting on an ensemble of particles. The boundary conditions associated with (10) are determined by the requirement that the probability flux must vanish at zero and infinity. Therefore, it is reasonable to consider the domain of the problem as the interval of $[1,5] \mu \mathrm{m}$ with zero flux at the boundaries.

The main difficulties associated with the advection problem for the myofibril are: (1) the velocity field is discontinuous. (2) there are stopping points, where the local velocity vanishes. (3) the velocity field is time-dependent through external constraints on the myofibril. These features are illustrated in Fig. 4. 


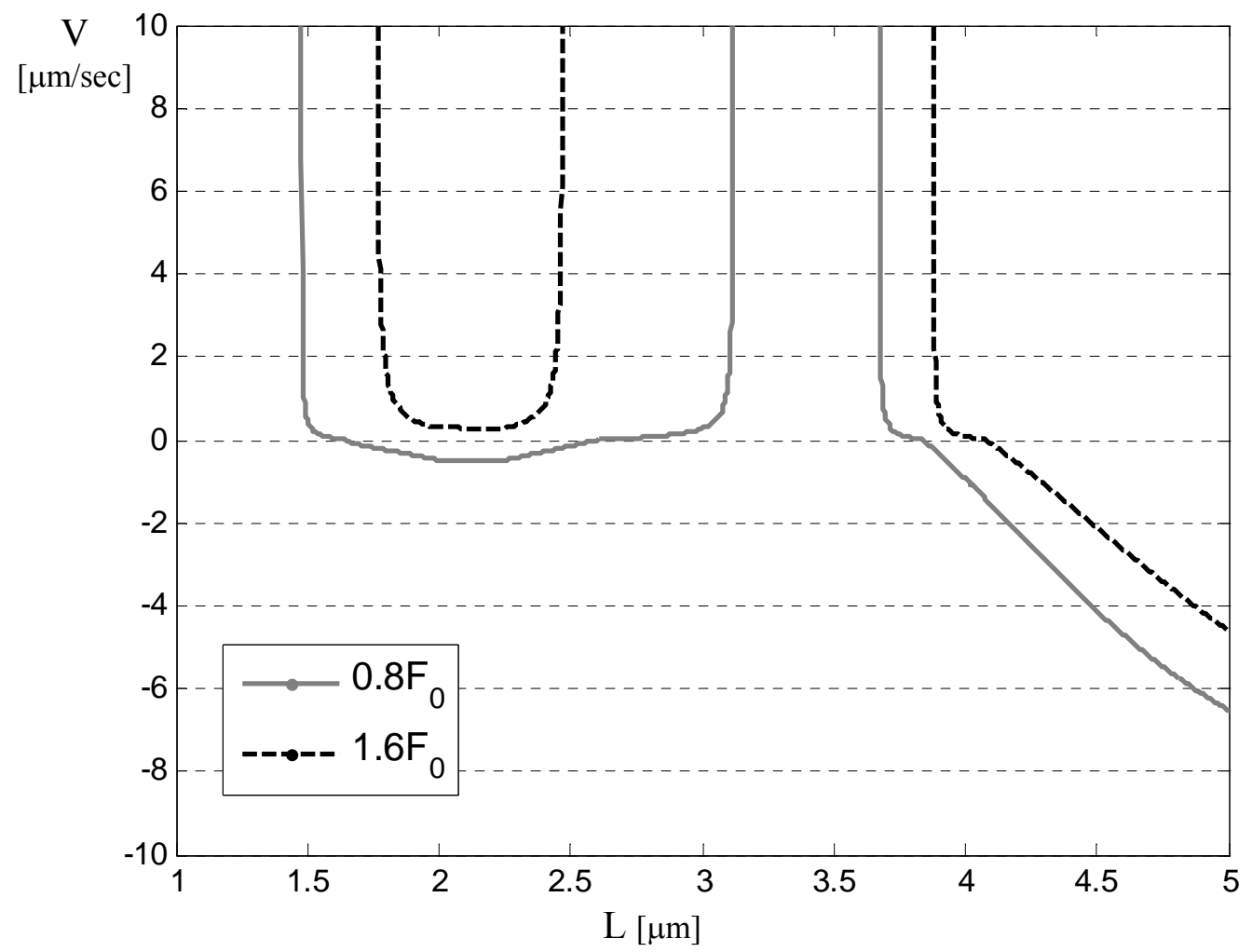

Fig. 4: Velocity as a function of sarcomere length at a fixed force. For the smaller force, there are two stable sarcomere lengths $\left(\mathrm{v}(\mathrm{L})=0, \mathrm{v}^{\prime}(\mathrm{L}) \leq 0\right)$ at 1.63 and $3.83 \mu \mathrm{m}$. There is only one stable sarcomere length $(\mathrm{L}=4.08 \mu \mathrm{m})$ for the higher force.

\subsection{Model F - stochastic temporal fluctuations}

There are stochastic temporal fluctuations in the force capacity of each sarcomere due to the inherent nature of the cross-bridge cycle and rapid fluctuations in Calcium ion concentration. Given the series architecture and negligible inertia, the temporal fluctuations in the force capacity of the sarcomeres translate to stochastic temporal fluctuations in their velocity (Iwazumi, 1987; Telley, et al., 2006). This can be modeled by adding a stochastic contribution, $\delta \mathrm{v}$, to the righthand-side of (6):

$$
\dot{L}^{(i)}=v\left(\frac{F^{(e x t)}(t)}{F_{1}\left(L^{(i)}\right)}\right)+\delta v^{(i)}
$$

Due to the large number of sarcomeres, these velocity fluctuations are independent (i.e. $\delta \mathrm{v}^{(\mathrm{i})}$ and $\delta \mathrm{v}^{(\mathrm{j})}$ are independent stochastic processes for $\mathrm{i} \neq \mathrm{j}$ ). Consequently, (11) may be viewed as a 
Langevin equation for L. Integration of (11) leads to the alternative formulation of a stochastic differential equation (Oksendal, 2003)

$$
d L(t)=V(t) d t+D W(t) \quad ; \quad V(t, L)=v\left(\frac{F_{\text {ext }}(t)}{F_{1}(L)}\right)
$$

Above, $\mathrm{W}(\mathrm{t})$ is a Wiener process, and $\mathrm{D}$ is a scalar constant that describes the underlying physics of the fluctuations.

Equations (11) and (12) represent a stochastic process. Nevertheless, the length distribution and its evolution in time are deterministic if the ensemble is large enough. The evolution in time of the length distribution is governed by the Fokker Planck (FP) equation, which has the form of (Caughey, 1963; Risken, 1989):

$$
\frac{\partial P(L, t)}{d t}=\left(-\frac{\partial}{d L} D_{1}(L, t)+\frac{\partial^{2}}{d L^{2}} D_{2}(L, t)\right) P(L, t) .
$$

$D_{1}$ and $D_{2}$ are termed drift and diffusion coefficients, respectively. The drift and diffusion coefficients are the first two members of the Kramers-Moyal expansion coefficients:

$$
D_{n(\bar{L}, t)} \equiv \frac{1}{n !} \quad \lim _{\tau \rightarrow 0} \frac{1}{\tau}<(L(t+\tau)-\bar{L})^{n}>\left.\right|_{L(t)=\bar{L}},
$$

where $<>$ denotes ensemble average. It can be shown (Risken, 1989) that for the process described by (12), these coefficient vanish for $\mathrm{n} \geq 3$. Furthermore,

$$
D_{1}=V(L, t) \quad ; \quad D_{2}=D
$$

Inserting these values into the FP equation (13), we conclude that the following partial differential equation governs the dynamics of the sarcomere length distribution:

$$
P_{, t}+(V P)_{, L}-D P_{, L L}=0 .
$$

Equation (16) describes a conservation law for the function $\mathrm{P}(\mathrm{L}, \mathrm{t})$. This can be seen by writing the equation in the form

$$
P_{, t}+J_{, L}=0 \quad ; \quad J \equiv V P-D \cdot P_{, L} .
$$


The "probability flux", J, defined in (17) includes two contributions. The first contribution is identical to the flux associated with Model H, (8). The second contribution governs the dispersion of $\mathrm{P}$ through the magnitude of the parameter $\mathrm{D}$.

As in Model $\mathrm{H}$, the probability flux vanishes at the boundaries $\mathrm{L}=1,5$.

\subsubsection{Estimation of the dispersion parameter $\mathbf{D}$}

The parameter D describes the physics of the underlying fluctuations. It is extremely difficult to estimate it directly. However, it is possible to infer it from the stationary solutions of (16). For stationary solutions, the probability flux must vanish. Hence, from (17), the differential equation for the stationary solution, $P_{\text {st }}$, is

$$
V(L) P_{s t}(L)-D \cdot \frac{d P_{s t}(L)}{d L}=0
$$

The solution of (18) is

$$
P_{s t}(L)=C \cdot \exp \left(\frac{1}{D} \int V(L) d L\right)
$$

where $\mathrm{C}$ is a constant of integration which has to be chosen such that $P_{\mathrm{st}}$ is normalized:

$$
\int_{0}^{\infty} P_{S t}(L) d L=1
$$

For this steady state solution, the average and standard deviation of the ensemble are

$$
\begin{aligned}
& \operatorname{Avg}\left(L_{s t}\right)=\left\langle L_{s t}\right\rangle=\int_{0}^{\infty} L P_{s t}(L) d L \\
& \operatorname{Std}\left(L_{s t}\right)=\left\langle\left(L_{s t}-<L_{s t}>\right)^{2}\right\rangle^{1 / 2}=\left(\int_{0}^{\infty}\left(L-<L_{s t}>\right)^{2} P_{s t}(L) d L\right)^{1 / 2} .
\end{aligned}
$$

A typical value for the standard deviation in sarcomere lengths for isometric contraction close to the optimal length is of the order of $0.05 \mu \mathrm{m}$. (Edman and Reggiani, 1984; Marland, 1998; Morgan, et al., 1982). This, along with (19) and (21), lead to a value of $\mathrm{D}=10^{-3} \mu \mathrm{m}^{2} / \mathrm{sec}$. The analytical solution, (19), associated with this value is illustrated in Fig. 5. 


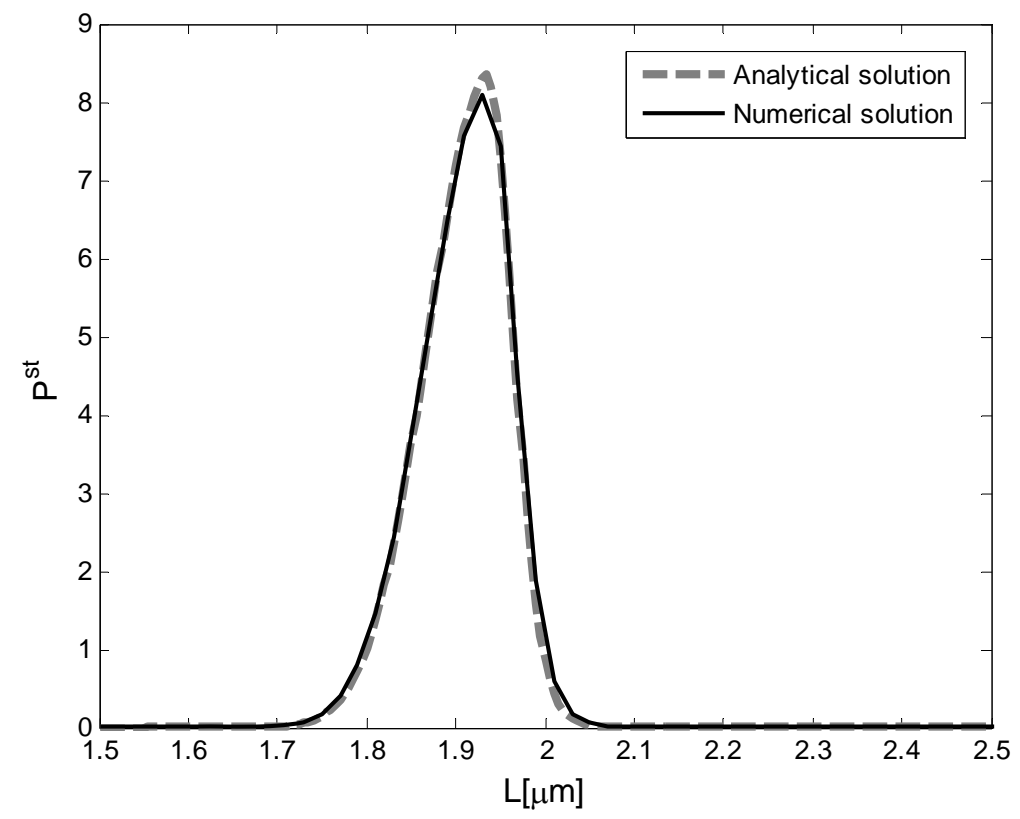

Fig. 5: Steady state solution of the Fokker-Planck equation (16) with $D=10^{-3} \mu \mathrm{m}^{2} / \mathrm{sec}$ and $\mathrm{F}_{\text {ext }}=1$. Comparison between analytical solution (19), and length distribution after 20 seconds of simulation using a high order finite volume numerical scheme.

\subsection{Model V - physiological variation}

The third model assumes that sarcomere length non-uniformities mainly stem from physiological variability in the mechanical properties of the sarcomeres. For example, variation in the myofibril diameter leads to different force capacities of sarcomeres, i.e., for the same length and velocity a sarcomere with larger diameter has more thin and thick filaments and thus produces a higher force. This can be modeled by introducing a force capacity parameter, C, into (1):

$$
F_{\text {ext }}=C^{(i)} \cdot F_{1}\left(L^{(i)}\right) \cdot \phi\left(\dot{L}^{(i)}\right)=\widetilde{F}_{1}^{(i)}\left(L^{(i)}\right) \cdot \phi\left(\dot{L}^{(i)}\right)
$$

for the i-th sarcomere. The distribution of the parameter $\mathrm{C}$ among the ensemble of sarcomeres describes the biological variability of the sarcomeres. We emphasize that the intent of the parameter $\mathrm{C}$ is to describe the overall effect of biological variability and not a specific phenomenon or mechanism. We assume that changes in the distribution of $\mathrm{C}$ can be neglected during the time scale of the experiment; for example, the number of thin and thick filaments in each sarcomere is unchanged during the course of a typical experiment. 


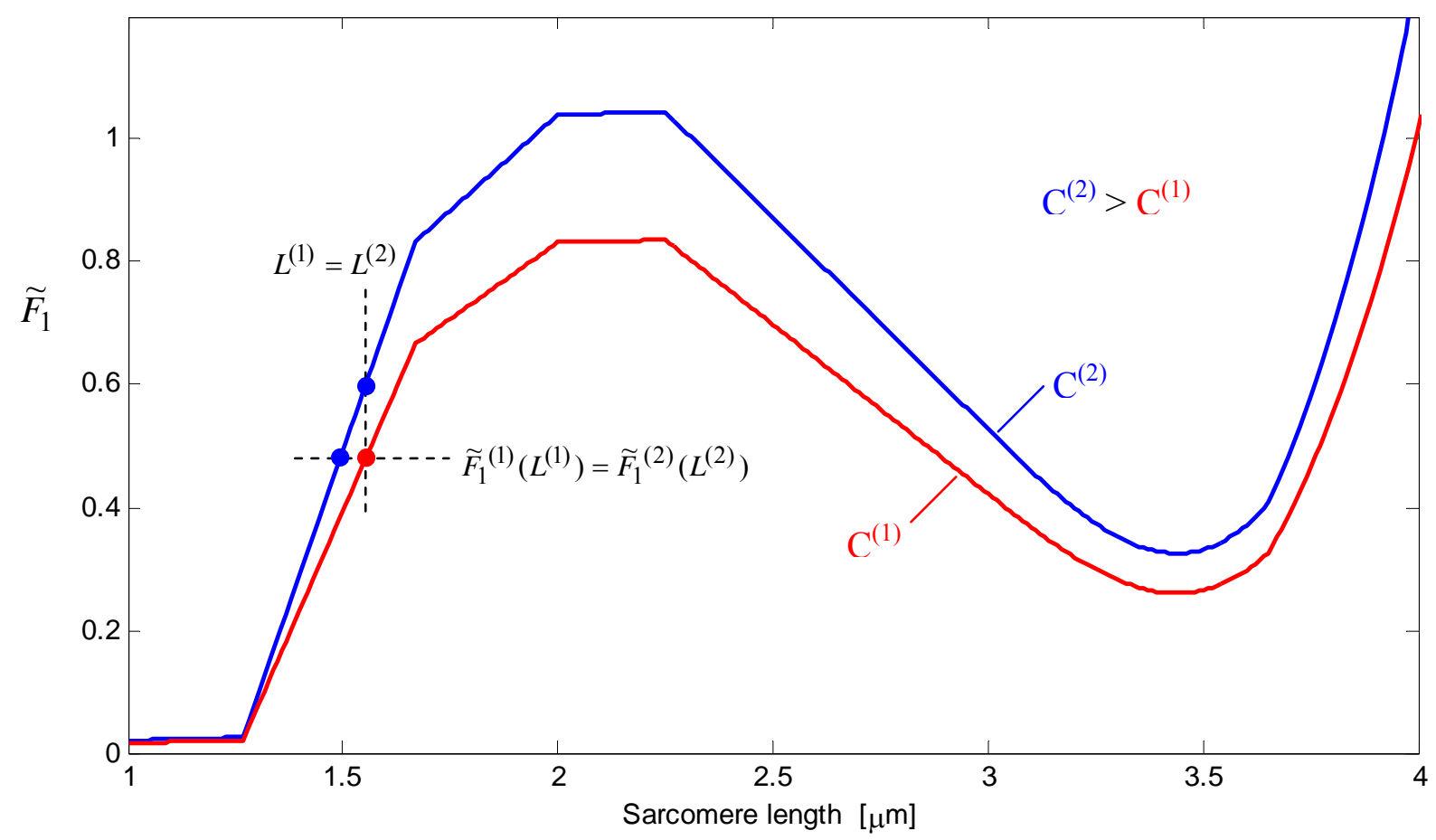

Fig. 6: Isometric force $F_{1}$ for two sarcomeres with a different value of force capacity factor $\mathrm{C}$. The isometric force generated by the weaker sarcomere, $\mathrm{C}^{(1)}$, is always smaller for the same length.

Consider two sarcomeres having different values of $\mathrm{C}, \mathrm{C}^{(1)}<\mathrm{C}^{(2)}$. The isometric force, $\widetilde{F}_{1}^{(i)}$, associated with these sarcomeres is illustrated in Fig. 6. Since $\mathrm{C}^{(1)}<\mathrm{C}^{(2)}$

$$
\widetilde{F}_{1}^{(1)}(L)<\widetilde{F}_{1}^{(2)}(L) \quad \forall L
$$

Hence, the first sarcomere is denoted as the "weaker", and the second the "stronger". From (22) it follows that

$$
\widetilde{F}_{1}^{(1)}\left(L^{(1)}\right) \cdot \phi\left(\dot{L}^{(1)}\right)=\widetilde{F}_{1}^{(2)}\left(L^{(2)}\right) \cdot \phi\left(\dot{L}^{(2)}\right) .
$$

Using relation (23), it follows that if $\mathrm{L}^{(1)}=\mathrm{L}^{(2)}$,

$$
\phi\left(\dot{L}^{(1)}\right)>\phi\left(\dot{L}^{(2)}\right) .
$$

Furthermore, since $\phi$ is a strictly monotonously increasing, we conclude that

$$
\dot{L}^{(1)}>\dot{L}^{(2)} \text {. }
$$


Before activation, the contraction force of the sarcomere is described by (1) with $\mathrm{F}_{\mathrm{A} 1}=0$. Furthermore, it is reasonable to assume that before activation the sarcomeres are at or close to static equilibrium, i.e.:

$$
F=C^{(i)} \cdot F_{T 1}(L)
$$

Since $\mathrm{F}_{\mathrm{T} 1}$ is a monotonously increasing function, we conclude that

$$
L^{(1)}(t=0)>L^{(2)}(t=0) \text {. }
$$

Together, equations (26) and (28) imply that the weaker sarcomere is longer for all times:

$$
L^{(1)}(t)>L^{(2)}(t) \quad \forall t
$$

This fact then has an important consequence. It implies that in a distribution of sarcomeres, the sarcomere in the $n$-th percentile in the length distribution is in the $n$-th percentile in the force capacity distribution and vice-versa. Consequently, we may write $C=C(L, P)$. Specifically, let $\mathrm{P}_{\mathrm{C}}$ describe the distribution function for the force capacity $\mathrm{C}$. Since $\mathrm{C}$ is physiological, $\mathrm{P}_{\mathrm{C}}$ is independent of time. Further, since the weaker sarcomeres are always longer, the sarcomere at the $n$-th percentile force capacity will be at the n-th percentile of the length distribution: $\int_{0}^{L} P(\ell) d \ell=\int_{C}^{\infty} P_{C}(c) d c$. This implicitly defines the relation $\mathrm{C}=\mathrm{C}(\mathrm{P}, \mathrm{L})$, which is illustrated schematically in Fig. 7.

We can now follow the line of argument in Section 3.1 for model $\mathrm{H}$ to conclude that for model $\mathrm{V}$, the length distribution evolves according to the following equation:

$$
\frac{\partial P(L, t)}{d t}+\frac{\partial}{d L}(P(L, t) V(L, t, C(L, P))=0
$$

Here, the velocity field is defined by

$$
V(L, t, C(L, P))=v\left(\frac{F_{\text {ext }}(t)}{C(L, P) F_{1}(L)}\right)
$$

Note that in contrast to (10), the flux now depends on $\mathrm{P}$, and (30) constitutes a nonlinear partial differential equation. Nevertheless, the fact (29) ensures that the characteristics of the partial 
differential equation (30) do not cross each other. Hence, shocks do not form, and the nonlinear conservation law has a unique weak solution (LeVeque, 1998).

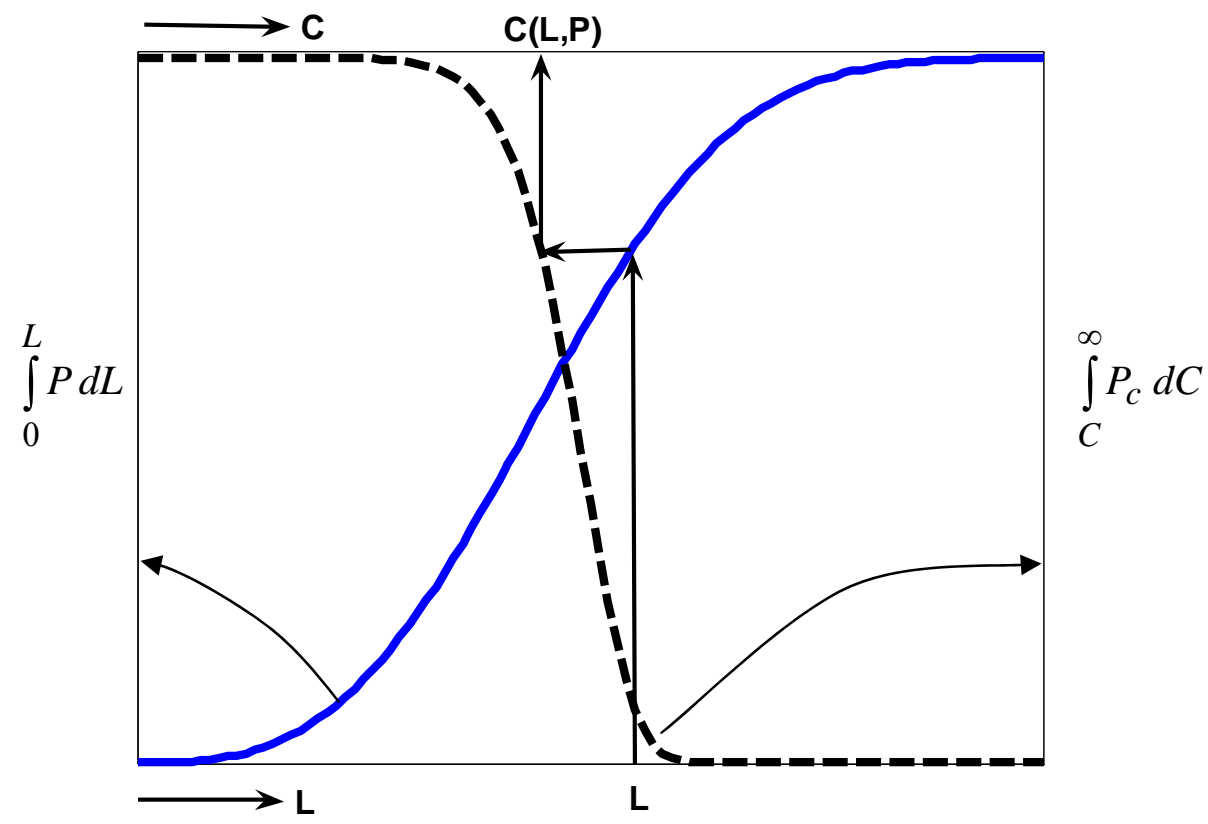

Fig. 7: A schematic description of the procedure for calculating the relation $C(L, P)$. $P_{C}$ describes the distribution function of $C$. Note that $P_{C}$ is time invariant while $P$, the sarcomere length distribution, is time dependent. The procedure is based on the fact that the sarcomere in the $n$-th percentile of the length distribution is in the $\mathrm{n}$-th percentile of the force capacity distribution. 


\section{Numerical procedure}

The numerical solution of the models presented in Section 3 requires care. First order finitedifference spatial (with respect to L) derivatives introduce substantial artificial (numerical) dissipation (LeVeque, 1998; 2002), and second order finite-difference spatial derivatives introduce large artificial oscillations, which may eventually become unbounded (LeVeque, 1998; 2002). Further, the flux is discontinuous, as in (10), and its spatial derivative has to be interpreted in the sense of distributions. Therefore, we use a finite volume numerical scheme with a high order reconstruction and Monotonized Central-Difference (MC) Limiter (LeVeque, 1998; 2002).

\subsection{Force control experiments}

In force control experiments, the external force at each given time is prescribed, and there are no geometrical constraints. We consider the spatial domain $[1,5] \mu \mathrm{m}$ of sarcomere lengths and impose no-flux boundary conditions. We begin with Model $\mathrm{H}$ described by the linear partial differential equation (10). We discretize time, and obtain the function $\mathrm{P}_{\mathrm{n}+1}$ at time step $\mathrm{n}+1$ based on $\mathrm{P}_{\mathrm{n}}$ as follows:

- Divide the length domain, the interval $[1,5] \mu \mathrm{m}$ of sarcomere lengths, into cells of uniform size.

- Calculate the average value of $P_{n}$ at each cell.

- Given the cell averages, reconstruct a local representation of the function that agrees with the average cell value. In the current work, a first order reconstruction (linear behavior within each cell) is adopted. Local slope at each cell is obtained by the MC limiter method.

- For each cell, calculate the "probability flux" from its boundaries: since the problem is linear with respect to $\mathrm{P}$, characteristics do not cross each other; thus, the solution and its characteristics are unique. Therefore, the flow at each cell boundary can be obtained by calculating the "reverse" characteristics

$$
\dot{L}=v\left(\frac{F_{\text {ext }}(t)}{F_{1}(L)}\right)
$$

using a fourth order Runge-Kutta method (see Fig. 8). 
- Given the inflow and outflow at the boundaries, the average values of $\mathrm{P}_{\mathrm{n}+1}$ are calculated.

- Time-step is limited by the CFL condition, which guarantees that the characteristics do not travel more than the size of one cell during a single time-step (LeVeque, 1998; 2002).

The numerical scheme adopted for solving the Fokker Planck equation (13) associated with Model F is similar to the one described above. The only difference is that the contribution of the diffusion term with $\mathrm{D}=10^{-3} \mu \mathrm{m}^{2} / \mathrm{sec}$ is added to the flux at the boundaries of each cell. The accuracy of the numerical scheme is verified in Fig. 5, where the "steady-state" solution of the numerical scheme is compared with the exact (analytical) steady state solution.

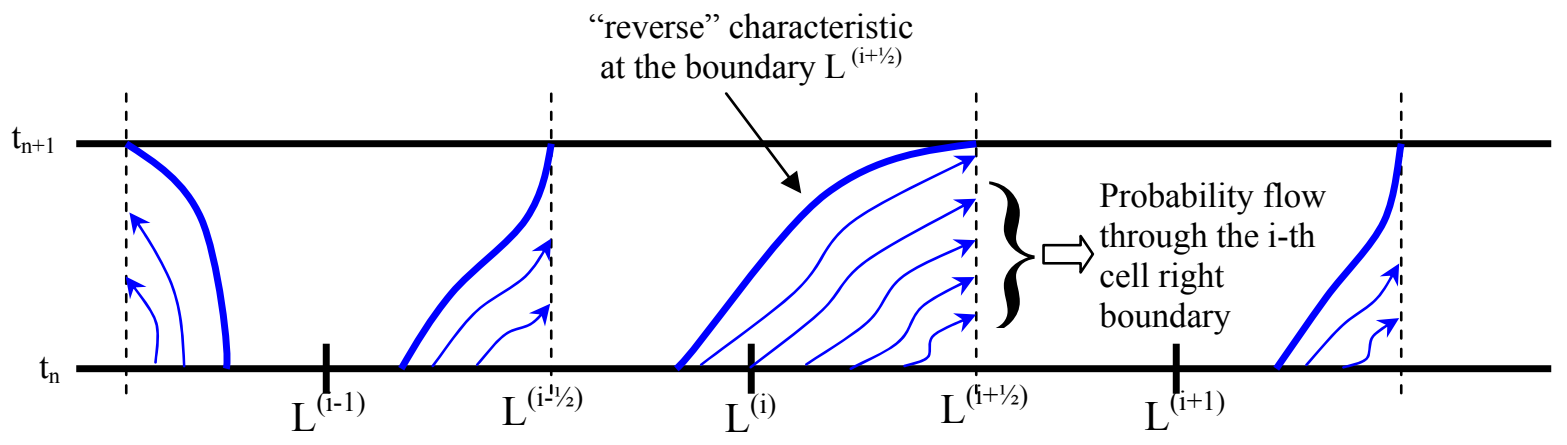

Fig. 8: Finite volume method: a schematic illustration of the cells and associated "reverse" characteristics at their boundaries. Arrows are used to illustrate probability flow through the cell boundary.

The partial differential equation (30) that governs Model V is nonlinear. Nevertheless, as discussed in Section 3.3, the characteristics associated with this equation do not cross each other. Therefore, (30) has a unique weak solution. Furthermore, a numerical scheme similar to the one used for solving Model $\mathrm{H}$ can be adopted. The only difference is that at each time step the value of $\mathrm{C}$ (the biological variability parameter) associated with the characteristics at the boundaries of each cell has to be calculated. This can be accomplished following the procedure illustrated in Fig. 7. Once the values of $\mathrm{C}$ at the cell boundaries are obtained, the corresponding characteristics are calculated by a $4^{\text {th }}$ order Runge-Kutta method, and the rest of the scheme is identical to the one used for solving Model $\mathrm{H}$. 


\subsection{Length control experiments}

Almost all experimental studies of the mechanics of muscles involve length control conditions where the ends of a muscle fiber (or a whole muscle) are attached to two grips, the relative distance between the grips is controlled, and the resultant force is measured. Therefore, the force and consequently the velocity field are not known a-priori. Instead, they have to be obtained by enforcing the constraint

$$
L_{\text {avg }}(t)=\int_{0}^{\infty} L \cdot P(L, t) d L
$$

where $\mathrm{L}_{\mathrm{avg}}$ is the prescribed length. We do so by guessing a force, solving the relevant equation following the procedure described in Section 4.1, and iterating over the force till the geometric condition (33) is satisfied.

\subsection{Cyclic force control}

For simulating cyclic load activities with many cycles using Models $\mathrm{H}$ and $\mathrm{V}$, we use a method that is similar in essence to the finite volume method, but updates $\mathrm{P}$ at the end of each cycle rather than at the end of each time-step. We begin by reconstructing a piecewise uniform representation that agrees with the average value of $\mathrm{P}$ in each cell at the beginning of the cycle. We calculate the characteristics associated with each cell boundary during one period using a fourth order Runge-Kutta method with a time step identical to that used in Section 4.1. We then reconstruct $\mathrm{P}$ at the end of one period using the fact that characteristics do not cross each other and that there is no flux across a characterisic.

We note that here the CFL condition does not necessarily hold since characteristics may travel more than one cell during a single time-step (cycle). Hence, the numerical procedure requires some care, and is made possible only due to the fact that characteristics do not cross. The advantage of this procedure is especially significant for Model $\mathrm{H}$, where the characteristics are independent of $\mathrm{P}$ and thus need to be calculated only once. We also note that the procedure is not adequate for Model $\mathrm{F}$ since the contribution of diffusion can not accounted for by this procedure. 


\section{Results}

\subsection{Experiments of Talbot and Morgan (1996)}

Talbot and Morgan (1996) provide quantitative information regarding the lengths of individual sarcomeres and their distribution. In their study, they applied length control protocols on toad muscles. At the end of a protocol, the muscle was "fixed" (Brown and Hill, 1982) such that the banding pattern was preserved, and sarcomere lengths were measured with the aid of electron microscopy. In what follows we simulate protocols used by Talbot and Morgan (1996) associated with rapid muscle stretches during tetanus (muscle is fully activated).

The first protocol involves eccentric contraction: A muscle with a 30mm "optimum length" was tetanized at $2 \mathrm{~mm}$ beyond optimum for $0.5 \mathrm{sec}$, and then stretched by $3 \mathrm{~mm}$ at a rate of 90 $\mathrm{mm} / \mathrm{s}$. At the end of stretch the muscle was fixed. A surface myofibril (surface myofibrils get fixed the fastest) was sectioned from the muscle and viewed under the electron microscope to observe the distribution of lengths of 1803 sarcomeres, all from same myofibril. One hundred and fifty four sarcomeres, which make up $8.5 \%$ of the population, were overstretched.

The above protocol, illustrated in the inset of Fig. 9a, was simulated by solving the governing equations associated with Models F (16) and V (30), under the geometrical constraint (33). Initial distribution was taken as Gaussian with 5\% standard deviation. The resultant force is shown in Fig. 9a. We divide the response into four phases: (1) A steep increase in force as a response to the sudden and rapid stretch. (2) A high level of force in order to withstand the rapid stretch followed by a gradual reduction as some sarcomeres begin to overstretch. The force required to stretch the fiber according to Model $\mathrm{V}$ is smaller compared to Model $\mathrm{F}$. The reason is that weaker sarcomeres overstretch under lower external forces. Therefore, overstretching begins earlier in Model V compared to Model F. (3) A steep decrease in force at the end of stretch. (4) A gradual increase in force while the overall length remains constant, "creep". 

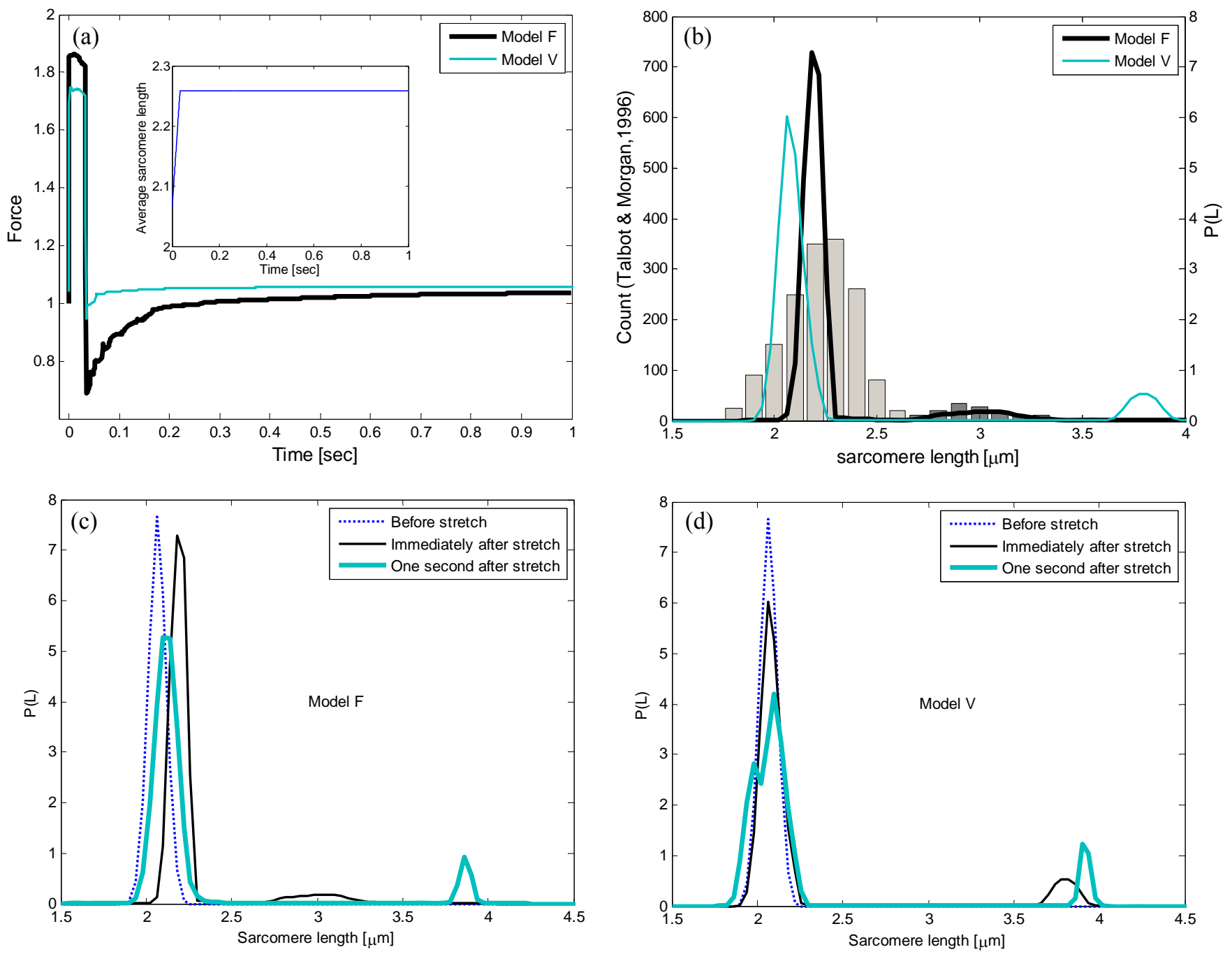

Fig. 9: Simulation of an eccentric contraction protocol studied by Talbot and Morgan (1996). (a) The resultant force. Inset: Imposed length vs. time. (b) Final distribution of sarcomere lengths and comparison between experimental observation (histogram, based on results of Talbot and Morgan (1996)) and the results of simulation. (c,d) Evolution in time of the length distribution predicted by Models F and V respectively.

A comparison between the results of the model and the experimental results of Talbot and Morgan (1996) is presented in Fig. 9b. It is evident that there is a good agreement between the two, especially with respect to the formation of the two separate populations of normal and overstretched sarcomeres. Indeed, both models predict $8.1 \%$ overstretched sarcomeres - very close to the value observed experimentally. This is important because no fitting parameters have been used in the analysis, and parameters defining dispersion and variation in the mechanical 
properties were inferred from independent experimental studies. The differences in the dispersion of the "normal" population and the lengths of the overstretched sarcomeres between the theoretical and experimental results is probably attributed to the fixation process used by Talbot and Morgan (1996) and differences between the mechanical properties of the toad muscle and those used in the theoretical model.

The creep phenomenon has been observed in numerous experimental studies and is commonly explained qualitatively by sarcomere lengths non-uniformities (Gordon, et al., 1966a; b; Lombardi and Piazzesi, 1990; Morgan, et al., 1982). Some of these studies even showed, by tracking the length of small segments along the muscle-fiber, that during "muscle-creep" some of the segments shorten on the expense of the lengthening of others. Fig. $9 \mathrm{c}$ and $9 \mathrm{~d}$ that show the evolution of the distribution of sarcomere lengths for Models $\mathrm{V}$ and $\mathrm{F}$ respectively demonstrate that this feature is captured well by our model.

The second protocol studied by Talbot and Morgan (1996) was identical to the one described above, except that a $30 \mathrm{~mm}$ muscle fiber was tetanized at $5 \mathrm{~mm}$ below the optimum length (see inset of Fig. 10a). Therefore, contrary to the first protocol, the average sarcomere length is always on the ascending limb in this protocol.

The resultant force of Model V is shown in Fig. 10a. The force predicted by Model F is similar to that of Model V, and is not shown in the figure for clarity. The final length distribution is shown in Fig. 10b. Observe that there is a fundamental difference between the responses predicted by the two models: Model V predicts that the dispersion of the lengths increases and even leads to overstretched sarcomeres, as observed by Talbot and Morgan (1996). On the other hand, Model F predicts a significant decrease in the sarcomere length non-uniformities. In Model $\mathrm{V}$ the variability in the mechanical properties leads to the condition where weaker sarcomeres always have a longer length. Consequently, longer sarcomeres are weaker and have to maintain a higher velocity to support the force. Thus, the dispersion in length increases. On the other hand, the mechanical properties of all sarcomeres in Model F are identical; thus, on the ascending limb, longer sarcomeres have higher isometric force capacity and therefore a lower velocity. Thus, the lengths become more uniformly distributed, contrary to observations. We conclude, therefore, that biological variability rather than stochastic fluctuations are responsible for the observed nonuniformities. 

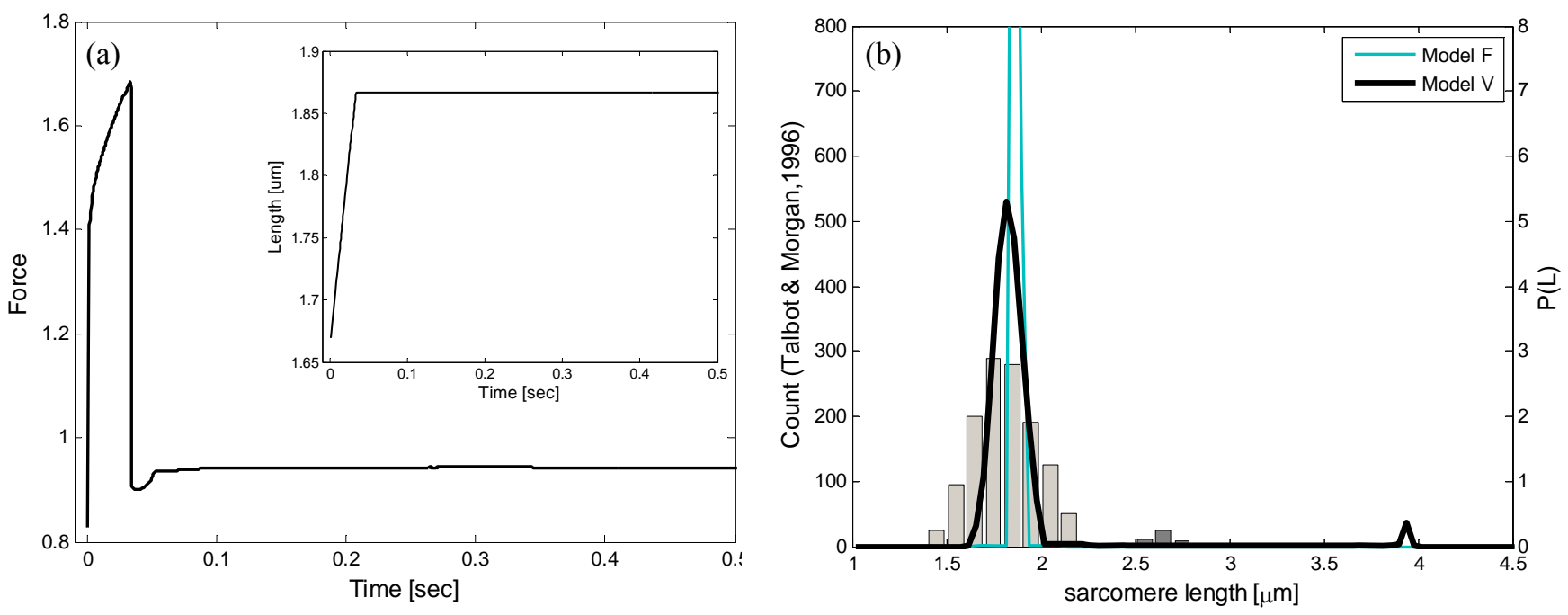

Fig. 10: Simulation of the eccentric contraction protocol on the ascending limb studied by Talbot and Morgan (1996). (a) The resultant force. Inset: Imposed length vs. time. (b) Comparison between experimental observations (histogram, based on results of Talbot and Morgan (1996)) and the results of simulation.

\subsection{Sarcomeres popping}

Sarcomere popping is a phenomenon hypothesized by Morgan and co-workers (Morgan, 1990; Morgan and Allen, 1999; Morgan and Proske, 2004; Talbot and Morgan, 1996) that involves a rapid increase of length of individual sarcomeres resulting in overstretching. This hypothesis is based on the widely-observed behavior of single muscle fibers (Katz, 1939; Lombardi and Piazzesi, 1990; 1992) where at moderate to high lengthening velocities the contractile force is approximately constant. However, recent experiments have questioned the popping hypothesis, though they have shown a clear evolution of length non-uniformities (Telley and Denoth, 2007; Telley, et al., 2006). We examine this in the context of our models.

Fig. 11 shows the results of a simulation of a myofibril that is initially at rest with $\mathrm{F}_{\text {ext }}=0.9 \mathrm{~F}_{0}$. The myofibril is subject to a rapidly increasing force that reaches $1.7 \mathrm{~F}_{0}$ and is held steady at that value (inset of Fig. 11a). We note that this protocol is not biologically meaningful, since muscles do not undergo such severe stretching even under the most extreme physiological conditions. Nevertheless, this protocol provides useful insight. Fig. 11a shows the accumulation of overstretched sarcomeres predicted by the three models, while Fig. 11b shows the evolution of length distribution for Model V. All sarcomeres eventually overstretch, irrespective of the model, though there are differences in the rates. Importantly, the length distribution has two sharp peaks 
at all times, with no observable sarcomeres between the peaks. This is despite the fact that all sarcomeres start at normal length and end up overstretched. The reason is that the transition from the normal length to the overstretched branch is very rapid. This means that it will be difficult to directly observe popping even when it occurs in an ensemble.
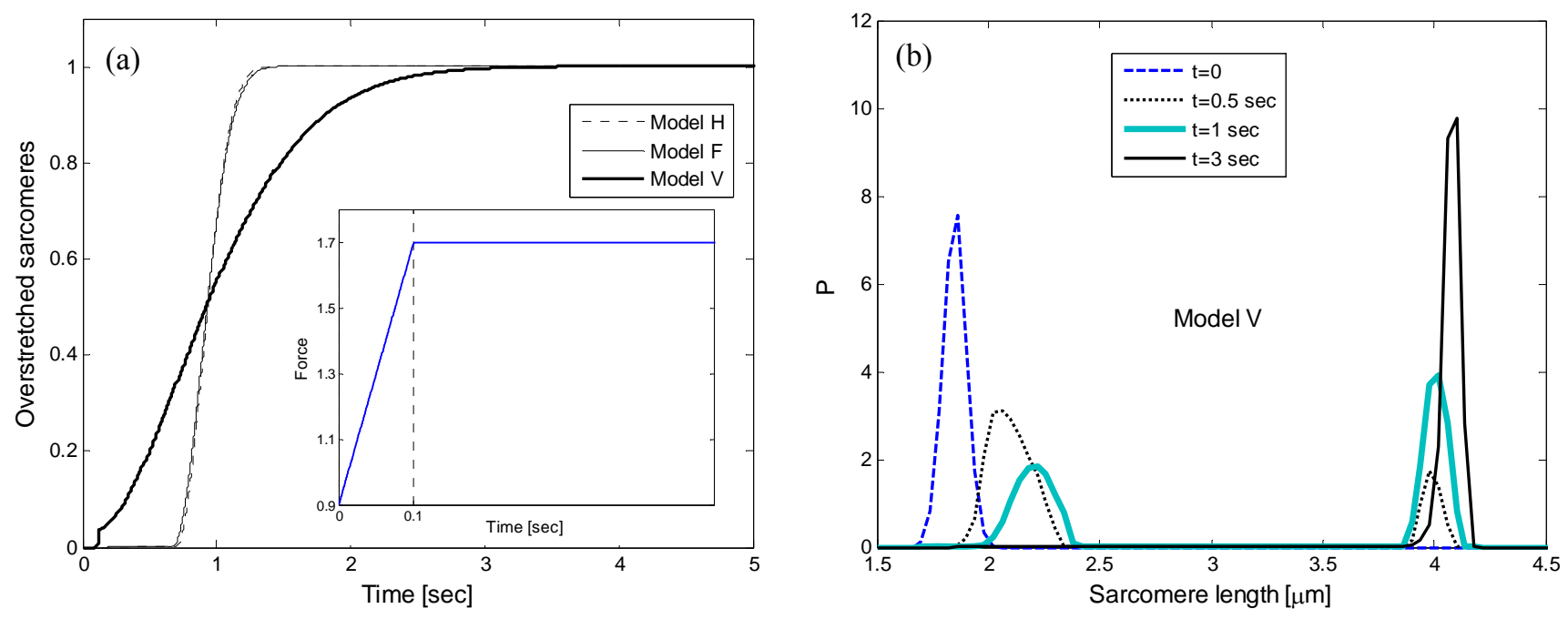

Fig. 11: Sarcomere popping due to an increase of the external force to $1.7 \mathrm{~F}_{0}$. (a) Accumulation of overstretched sarcomeres. inset: external force exerted on the myofibril. (b) Evolution of the length distribution for Model $\mathrm{V}$.

Further, we argue that popping is a stochastic effect that requires a very large number of sarcomeres subjected to a rapid stretch. Consider the limit case of only one sarcomere that undergoes a rapid and short (a few percents) length controlled stretch. Since the stretch is small and the sarcomere is length controlled, popping can not occur. Consider now 2 sarcomeres. If the total stretch is of only few percents, it is clear that it is not possible that both sarcomere pop. Further, since shortening is slow, popping of one sarcomere at the expense of shortening of the other is also not possible. However, if the number of sarcomeres is very large, a single sarcomere can increase its length rapidly at the expense of small and slow shortening of all other sarcomeres. To this extent, the popping hypothesis and the experiments of Telley and Denoth (2007) and Telley et al. (2006) can be reconciled.

\subsection{Sarcomeres homogenization}

We have seen that the bistability of the force-length relation can lead, under certain conditions, to the formation of two separated populations of normal and overstretched sarcomeres. Sarcomere 
homogenization (Denoth, et al., 2002) is the phenomena associated with the reunion of the two populations due to a long and significant decrease in the external force. Fig. 12 describes the homogenization process for an ensemble that is composed of approximately $10 \%$ overstretched sarcomeres and 90\% normal sarcomeres, see Fig. 12a. The external force imposed on the myofibril is illustrated in the inset of Fig. 12a. The homogenization process is clearly observed in Fig. 12b which shows the gradual decrease in the number of overstretched sarcomeres. The overall length response of the myofibril due to the imposed external load is shown in Fig. 12c. It can be divided into the following stages: (1) rapid length decrease due to the rapid decrease in force, which is associated with a drift of the overstretched population down the stable branch; (2) slower length changes as the overstretched population approach the minimum point of the isometric force, around sarcomere length of 3.5 micron; and (3) fast decrease in the myofibril length as the sarcomeres shorten on the descending limb. As the external force is increased again to its original level, $0.75 \mathrm{~F}_{0}$, the entire sarcomere population elongates in order to accommodate the higher force level. Although the force at the beginning and at the end of the simulation is identical, final length of the myofibril is significantly smaller compared to its original length. This is a direct result of the sarcomeres homogenization. We also note that the response associated with Model V differs from the response of the other two models, due to the same reason discussed in Section 5.2.

Fig. 12d shows the evolution of the length distribution associated with model V. For clarity, only the overstretched population is shown. Contrary to the popping phenomenon, a significant number of sarcomeres can be found on the descending limb. The reason is that the maximal shortening velocity of the sarcomere is much smaller compared to the velocity during popping (lengthening), see Fig. 3. 

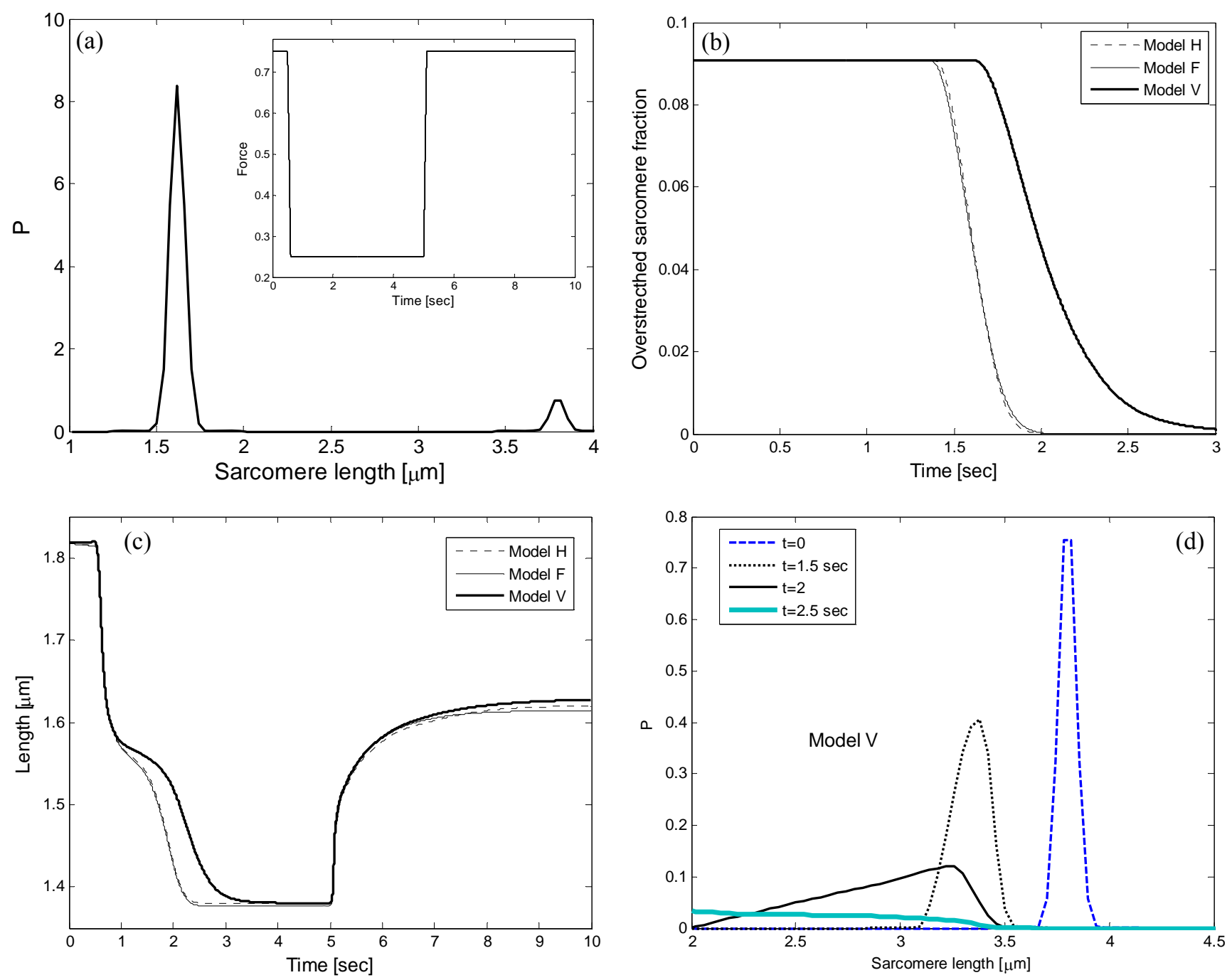

Fig. 12: Force controlled stretch-release experiment followed by force recovery after 5 seconds: (a) Initial distribution. Inset: external force vs. time. (b) Fraction of overstretched sarcomeres (c) Average sarcomere length (proportional to the overall myofibril length) (d) Evolution in time of the length distribution associated with Model V.

The next example, the results of which are displayed in Fig. 13, is identical except for a small change in the external load sequence: the recovery of the force to its original value takes place after 2 seconds instead of 5 (see Fig. 13a). As in the previous example, the decrease of the external force leads to shortening of the overstretched sarcomeres. However, contrary to the previous example, the early recovery of the force does not permit the sarcomere homogenization process to complete, and the final length of the myofibril is significantly different compared to the previous example. It should be noted that although the sarcomere homogenization process was not complete, a small fraction of the overstretched sarcomeres did pass to the normal length 
region. This can be seen by the fact that the final length of the myofibril is smaller than its original length.

The results of these two examples agree with the detailed simulations of Denoth et al. (2002). Nevertheless, a direct quantitative comparison is impossible due to the fact that their simulations involve only 12 sarcomeres connected in series, out of which 11 have normal length.
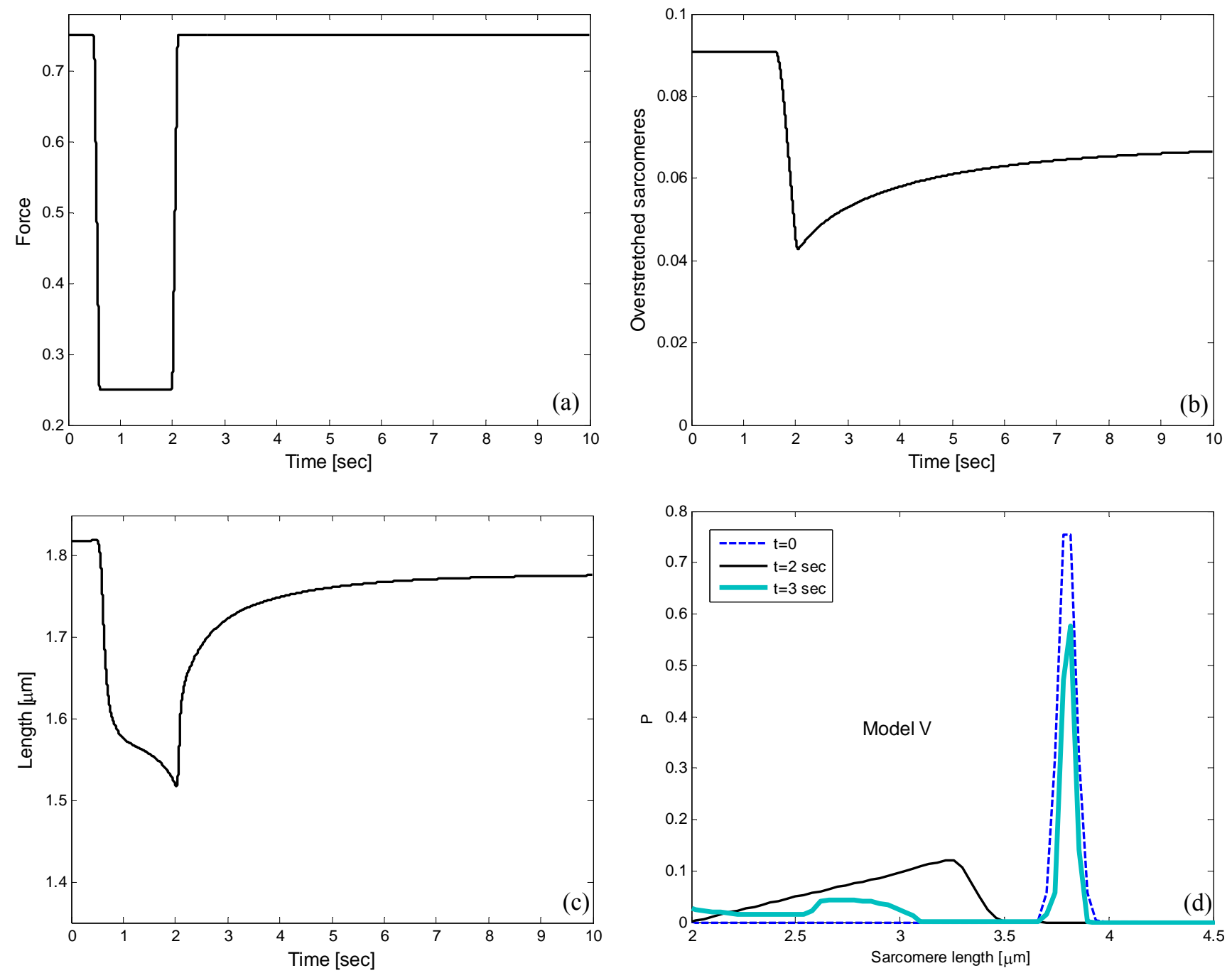

Fig. 13: Force controlled stretch-release experiment followed by force recovery after 2 seconds: (a) External force vs. time. (b) Fraction of overstretched sarcomeres (c) Average sarcomere length (proportional to overall myofibril length) (d) Evolution in time of the length distribution associated with Model V. 


\subsection{Cyclic loading}

Many daily activities involve eccentric contraction (active lengthening of the muscle) and cyclic loading. Simple examples include the experience the knee extensors during running downhill, skiing, and riding a horse. These activities differ in their magnitude and frequency, and may lead to muscle damage. The accumulation of muscle damage during eccentric contraction is directly related to the number of overstretched sarcomeres (Morgan and Allen, 1999; Morgan and Proske, 2004). Therefore, understanding the response of a muscle undergoing a cyclic load is important, and in particular understanding the effects of the load characteristics, such as the load frequency and magnitude, on the evolution of overstretched sarcomeres.

The next two sections include numerical simulations of a myofibril subjected to cyclic length control or force control conditions, respectively, using Model V. We note that real physiological cyclic activities involve cyclic activation and relaxation of the muscle synchronized with a cyclic load. Our current model does not incorporate activation, and assumes that the myofibril is constantly activated. Nevertheless, the results provide insight with respect in-vivo muscle damage.

\subsubsection{Length control}

We study the response of a myofibril to an imposed sinusoidal average length with a period of one second and a minimal length of 2 microns, see inset of Fig. 14b. Fig. 14a shows the accumulation of overstretched sarcomeres for two different amplitudes, $A=0.1 \mu \mathrm{m}$ and $\mathrm{A}=0.2 \mu \mathrm{m}$. The resultant force is illustrated in Fig. 14b.

We note that the average length is always normal and does not reach the descending limb of the force-length relation. Further, the stretch rate is not high, so sarcomere popping is not expected. Indeed, there is no accumulation of overstretched sarcomeres during the first cycle. However, overstretched sarcomeres begin to form after a few cycles and continue to accumulate for some time until the process reaches a steady state. The final number of overstretched sarcomeres as well as their accumulation rate increases with the amplitude of the overall length.

The force response of the myofibril becomes periodic when the accumulation of overstretched sarcomeres reaches a steady state (not shown in the figure). Interestingly, although the imposed overall length is sinusoidal, the "steady state" periodic force response is not. In particular, the 
increase in force during lengthening is significantly higher than the decrease in force during shortening.
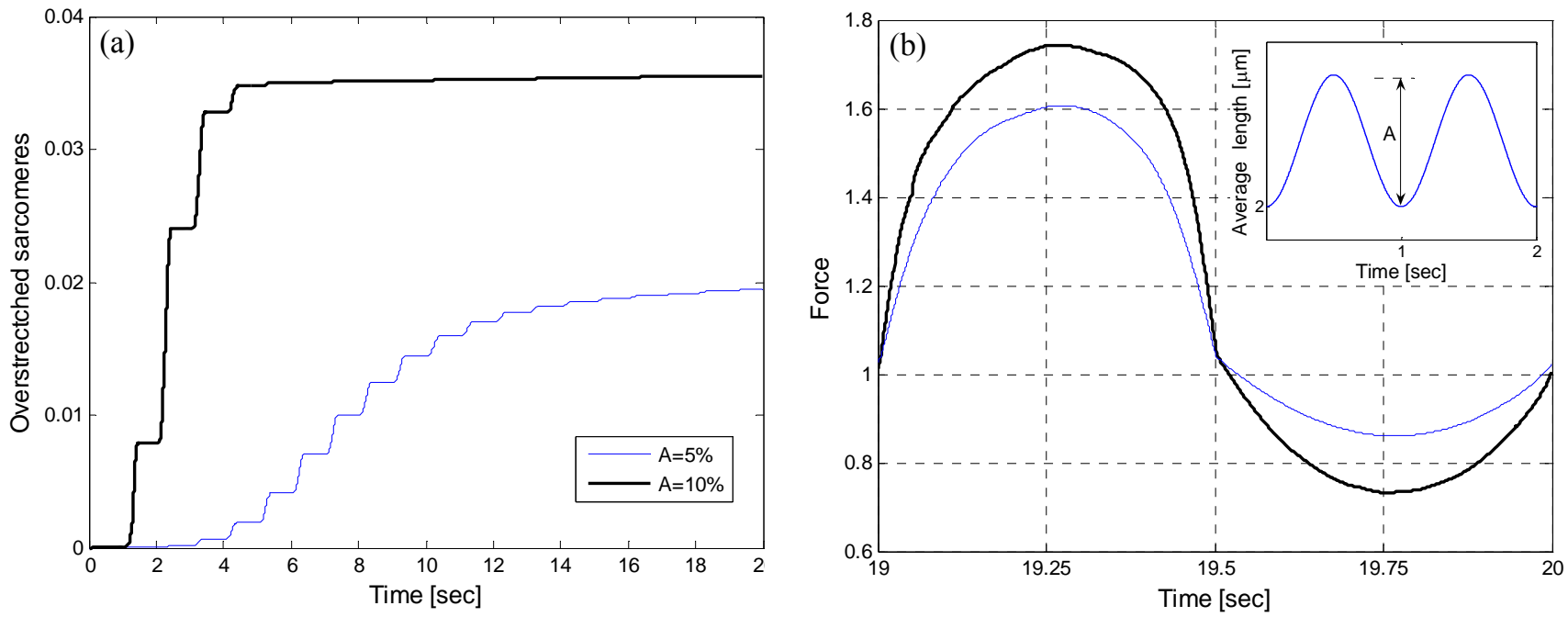

Fig. 14: Myofibril response to periodic length control. (a) Accumulation of overstretched sarcomeres. (b) Force response after 20 cycles. Inset: Schematic illustration of the imposed cyclic length.

\subsubsection{Force control}

Calculating the myofibril response for length control conditions is computationally expensive, especially if we are interested in the response over long times. Therefore, we study the response of a myofibril subjected to a cyclic load, and impose a force similar to the one obtained for length control conditions, Fig. 14b. In particular, the periodic load is assumed to be composed of two sinusoidal functions with the same period but different amplitudes (identical amplitudes lead to a sinusoid), see inset of Fig. 15b. We note that this form is merely a qualitative approximation for the force calculated in the previous section.

Fig. 15a and Fig. 15b show the accumulation of overstretched sarcomeres for a myofibril subjected to a cyclic load characterized by $f_{\min }=1$ and $f_{\max }=1.45$. The value of $f_{\min }=1$ is chosen to simulate a load similar to the one expected under physiological conditions. For example, during horse riding the knee extensors absorb the riding energy by actively stretching (force is higher than one) and then shorten as fast as possible (force equals one) in order to get back to the optimal "ready" length. Fig. 15c compares the accumulation of overstretched sarcomeres for 
different values of $f_{\max }$. The role of $f_{\min }$ is demonstrated in Fig. 15d which shows the fraction of overstretched sarcomeres after 10,000 cycles as a function of $\mathrm{f}_{\text {min }}$.
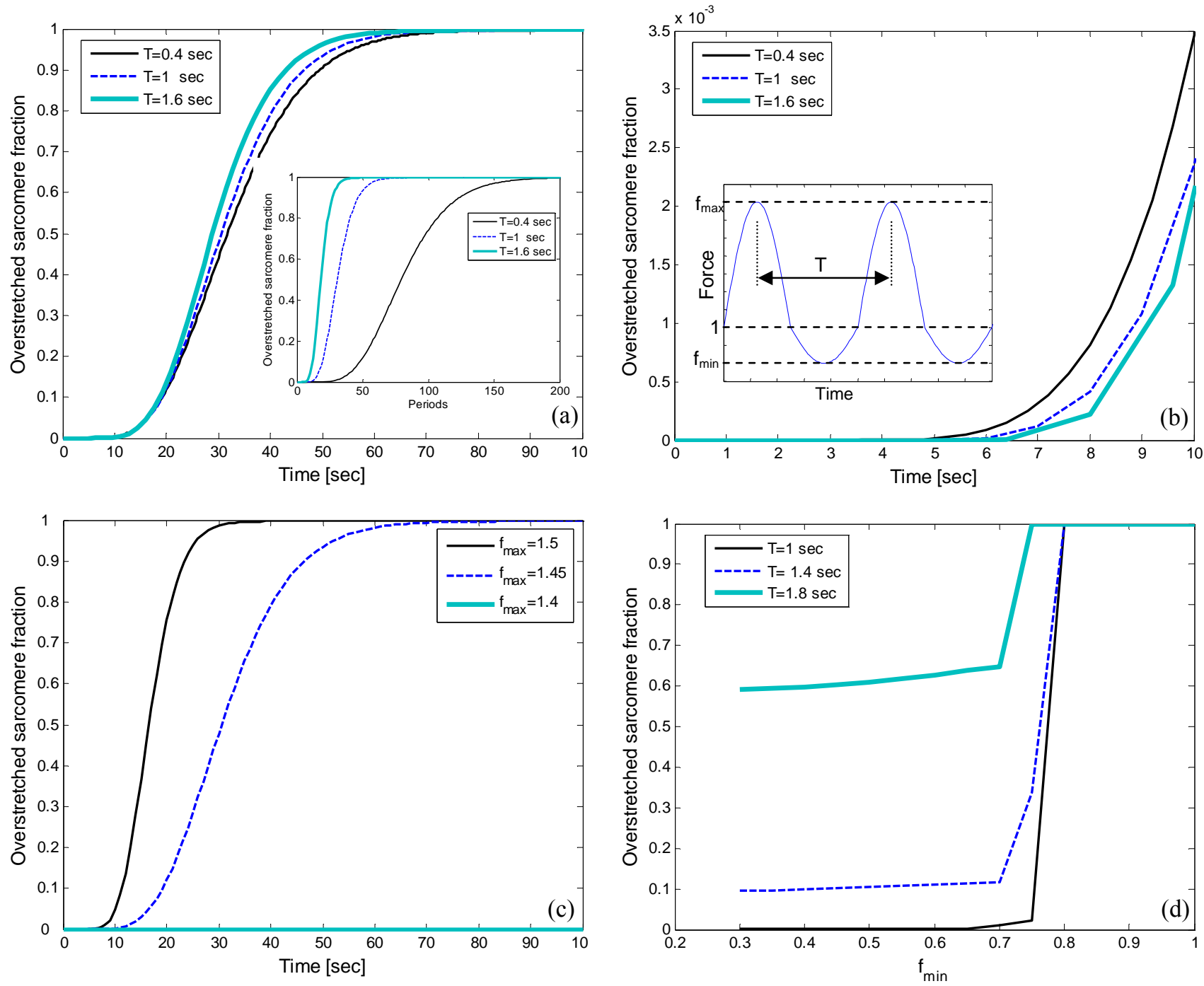

Fig. 15: Fraction of overstretched sarcomeres for a myofibril subjected to a cyclic force. (a) Accumulation of overstretched sarcomeres vs. time for different load frequencies with $\mathrm{f}_{\min }=1, \mathrm{f}_{\max }=1.45$. Inset: Accumulation of overstretched sarcomeres vs. number of cycles. (b) Accumulation of overstretched sarcomeres in the first 10 seconds for $f_{\min }=1$ and $f_{\max }=1.45$. Inset: Schematic illustration of the imposed cyclic force. (c) Accumulation of overstretched sarcomeres for different values of $f_{\max }$ and $f_{\min }=1, T=1$ second. (d) The fraction of overstretched sarcomeres after 10,000 cycles as a function of $f_{\min }$.

The frequency of the load has a significant effect on the accumulation of overstretched sarcomeres. Higher frequencies lead to more muscle damage (overstretched sarcomeres) in the first few seconds. Interestingly, the trend is opposite for long times. Further, we note that we can have overstretched sarcomeres with cycling, yet none in the first few cycles, as observed for 
length control conditions. The accumulation of overstretched sarcomeres is very sensitive to the value of $\mathrm{f}_{\max }$, and even a change of one percent can lead to dramatic effects. In particular, a small increase in $f_{\max }$ can increase the rate of accumulation of overstretched sarcomeres by a factor of 2. On the other hand, a slight decrease can lead to no accumulation at all. The value of $f_{\min }$ has also a dramatic effect on the fraction of overstretched sarcomeres. For small values of $f_{\min }$, the final fraction of overstretched sarcomeres (after many cycles) can have a wide range of values depending on the frequency of the load, but it is almost independent of $f_{\min }$. However, above a certain value of $f_{\min }$, the entire population becomes overstretched, independent of the load frequency.

\section{Summary}

The paper presents a new approach for studying the dynamics of a myofibril based on the collective behavior of physiologically relevant number of sarcomeres in the presence of stochastic fluctuations and biological variability. Contrary to current models which track (or solve for) each and every sarcomere, the proposed model directly describes the evolution of the sarcomere lengths distribution. This requires significantly smaller computational effort, and enables - for the first time - a study of physiologically-relevant ensembles of sarcomeres. The approach provides important insights and intuition. Further, it provides a more general framework, and is a first step towards bridging the micro (sarcomere) and macro (muscle) scales. Indeed, muscle fibers are made of a bundle of myofibrils. Since the interaction between sarcomeres on different myofibrils is small, the myofibrils in a muscle fiber are only constrained by the overall length. Hence, the analysis based on the proposed model with length control conditions is applicable to muscle fibers as well.

The model was validated by a quantitative comparison to experimental observations of sarcomere length distributions after various length control protocols. No fitting parameters were used, and mechanical properties as well as other parameters were inferred from independent experimental studies. The model is able to predict phenomena such as muscle creep, permanent extra tension, and length redistribution, and also provides quantitative insights about these phenomena for physiologically relevant number of sarcomeres. The results presented here motivate new experiments. Further, the analysis is not limited to a specific model of the single sarcomere, and the approach can be extended to other single sarcomere models. 
There is a fundamental difference between Models F (fluctuations) and V (variability). A comparison of the predictions of the models with reported quantitative measurements of sarcomere lengths distributions following various contraction protocols of fully activated muscle fibers show a very good agreement with Model V, while only partial agreement with model F. These results suggest that it is more likely that the main source for sarcomere non-uniformities is biological variability rather than rapid fluctuations in the force capacity of the sarcomeres.

We note that the value of the diffusion coefficient associated with Model F, $\mathrm{D}=10^{-3}\left[\mu \mathrm{m}^{2} / \mathrm{sec}\right]$, was obtained by fitting to observed dispersion $(\mathrm{W}=0.1 \mu \mathrm{m})$ under steady state isometric conditions. Interestingly, the relaxation time to reach steady state according to Model $\mathrm{F}$ is roughly $\mathrm{W}^{2} / \mathrm{D}=10$ seconds, which is much larger than the observed one (1-3 seconds). This inconsistency supports our conclusion that fluctuations are not the main source for sarcomere length non-uniformities.

One application of the model is related to muscle damage during eccentric contraction, which is directly related to the accumulation of overstretched sarcomeres. The proposed analysis can be used as a basis for developing "damage models". We note that almost all daily activities that involve cyclic loading include relaxation and activation of the muscle during each period. The current model does not consider activation, and including this is a natural next step.

The results of the model show that the overall behavior of the myofibril is dominated by sarcomere non-uniformities. This implies that deducing the behavior of a single sarcomere by measuring the response of a myofibril (and obviously muscle fiber) can lead to significant errors, and is subject to subjective interpretation. Since current experimental knowledge on the mechanics of the single sarcomere stems from single fiber experiments, the above conclusion motivates experiments on the level of single sarcomeres to validate the interpretation of these studies.

\section{Acknowledgements}

This work was carried out when Sefi Givli held a position at the California Institute of Technology. We thank a reviewer for the insightful comment on the typical relaxation time according to Model F. We are grateful to the National Science Foundation (Grant DMS-060667), the Powell Foundation and the Lester Deutch Foundation (Post-doctoral fellowship) for their financial support. 


\section{Appendix A: The single sarcomere model}

It is widely accepted (Denoth, et al., 2002; Morgan, et al., 1982; Telley, et al., 2006) that the mechanics of the single sarcomere is governed by three major contributions as illustrated in Fig. A.1: (A) an active force component, associated with the interaction between the thin and thick filaments, through the force generating cross-bridge action, (E) an elastic element, representing the elasticity of the thin and thick filaments, and (T) a passive force component, which represents the overall contribution of all passive elements in the sarcomere but is dominated by the titin molecule.

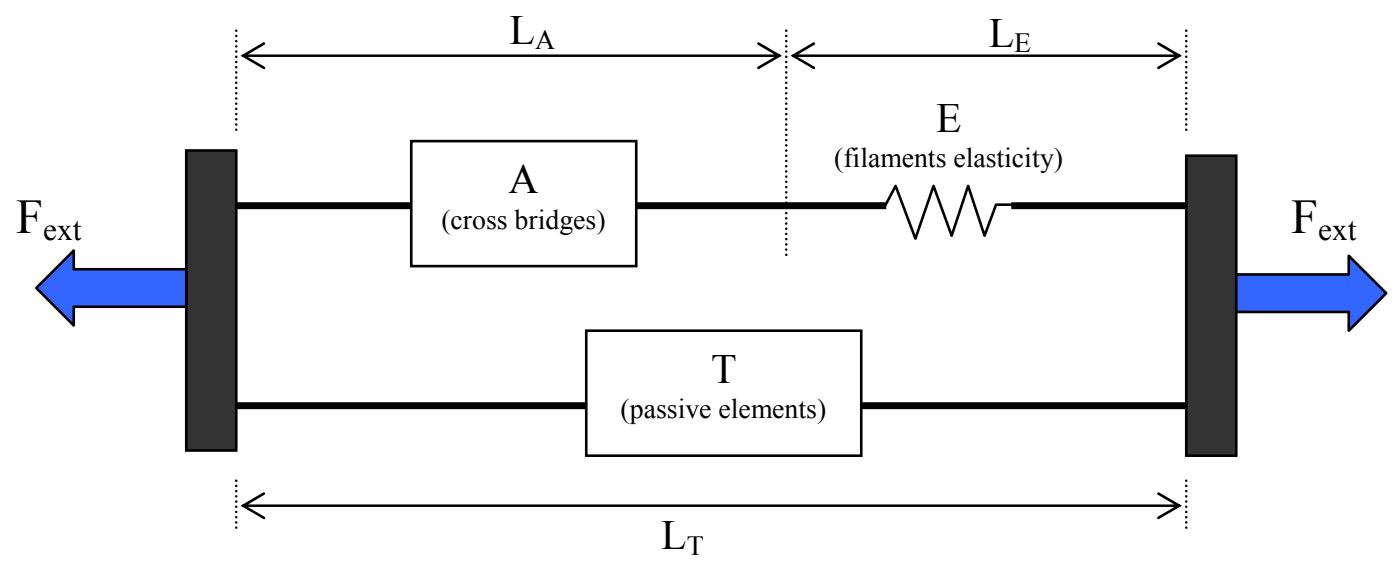

Fig. A.1: A lumped model of a single sarcomere following Denoth et al. (2002)

The forces generated by each of the sarcomere components depend both on the strain (length) and strain-rate (velocity) that the component experience. For specificity, we adopt the model of Denoth et al. (2002) in accordance with published experimental observations.

\section{A.1. Details of the sarcomere model derived by Denoth et al. (2002)}

Denoting $\mathrm{F}_{\mathrm{i}}, \mathrm{L}_{\mathrm{i}}(\mathrm{i}=\mathrm{A}, \mathrm{E}, \mathrm{T})$ as the forces and lengths associated with the active, elastic and passive components respectively, the force generated by the active component is

$$
F_{A}\left(L_{A}, \dot{L}_{A}\right)=F_{A 1}\left(L_{A}\right) \cdot \phi_{A}\left(\dot{L}_{A}\right)
$$


where $\mathrm{F}_{\mathrm{A} 1}$ is defined by a polygon with the data pairs in Table 1, extrapolated from Gordon et al. (1966a), and

$$
\phi_{A}\left(\dot{L}_{A}\right)=\left\{\begin{array}{ll}
1-1.2 \frac{\dot{L}_{A}}{\dot{L}_{A}-2} & \dot{L}_{A}<0 \\
1+0.8 \frac{2}{\pi} \arctan \left(6 \dot{L}_{A}\right) & \dot{L}_{A} \geq 0
\end{array} .\right.
$$

( ) denotes the time derivative. The lengths are measured in $\mu \mathrm{m}$, and forces are normalized by the maximal isometric force.

\begin{tabular}{|c|c|c|c|c|c|c|}
\hline $\mathrm{F}_{\mathrm{A} 1}$ & 0 & 0.8 & 1 & 1 & 0.005 & 0 \\
\hline $\mathrm{L}_{\mathrm{A}}[\mu \mathrm{m}]$ & 1.27 & 1.67 & 2 & 2.25 & 3.65 & 4 \\
\hline
\end{tabular}

Table A.1: Data pairs of the polygon defining $\mathrm{F}_{\mathrm{A} 1}\left(\mathrm{~L}_{\mathrm{A}}\right)$

The force-length relation, $\mathrm{F}_{\mathrm{A} 1}$, is a direct consequence of the filaments overlap (Gordon, et al., 1966a). On the other hand, $\phi_{\mathrm{A}}$ is a non-dimensional factor that reflects the effect of the relative velocity between the thin and thick filaments on the number of cross-bridges and the average cross-bridge force for a given filaments overlap. It should be noted that there is a wide agreement about the nature of the force-velocity relation, $\phi_{\mathrm{A}}$, for negative velocities or when the sarcomere shortens (Gordon, et al., 1966b; Hill, 1938; Katz, 1939). However, the force-velocity relation under eccentric contraction conditions, i.e. when the muscle is forced to lengthen while activated, is still under debate. This is true especially for relatively high lengthening velocities since sarcomere length non-uniformities appear almost immediately upon active lengthening. As long as the fiber lengthening is slow enough, these effects can be accounted for (Katz, 1939; Lombardi and Piazzesi, 1990; 1992). However, in order to measure the force-velocity relation associated with high sarcomere velocities, experiments on the scale of muscle fibers or even fiber segments can not work. Obviously, an experiment on a single sarcomere can be the solution, but the difficulties associated with such a set up are clear. Nevertheless, based on the available experimental data, a reasonable model for the force-velocity relation is the one given by (A.2).

The force associated with the passive component is 


$$
F_{T}\left(L_{T}, \dot{L}_{T}\right)=F_{T 1}\left(L_{T}\right) \cdot \phi_{T}\left(\dot{L}_{T}\right)
$$

with

$$
\begin{aligned}
& F_{T 1}\left(L_{T}\right)=\left\{\begin{array}{ll}
0 & \left\{\begin{array}{l}
L_{T}<0 \\
0.02
\end{array}\right. \\
0.02 L_{T}+1.2\left(L_{T}-3\right)^{3} & 0 \leq L_{T} \leq 3 \\
L_{T}>3
\end{array} .\right. \\
& \phi_{T}\left(\dot{L}_{T}\right)=\frac{2}{\pi} \arctan \left(2 \dot{L}_{T}\right)+1
\end{aligned}
$$

Also, the force of the elastic element is characterized by

$$
F_{E}\left(L_{E}\right)=\left\{\begin{array}{cc}
0 & L_{E}<0 \\
56 L_{E} & L_{E} \geq 0
\end{array} .\right.
$$

The forces associated with the sarcomere components are also illustrated in Fig. A.1.
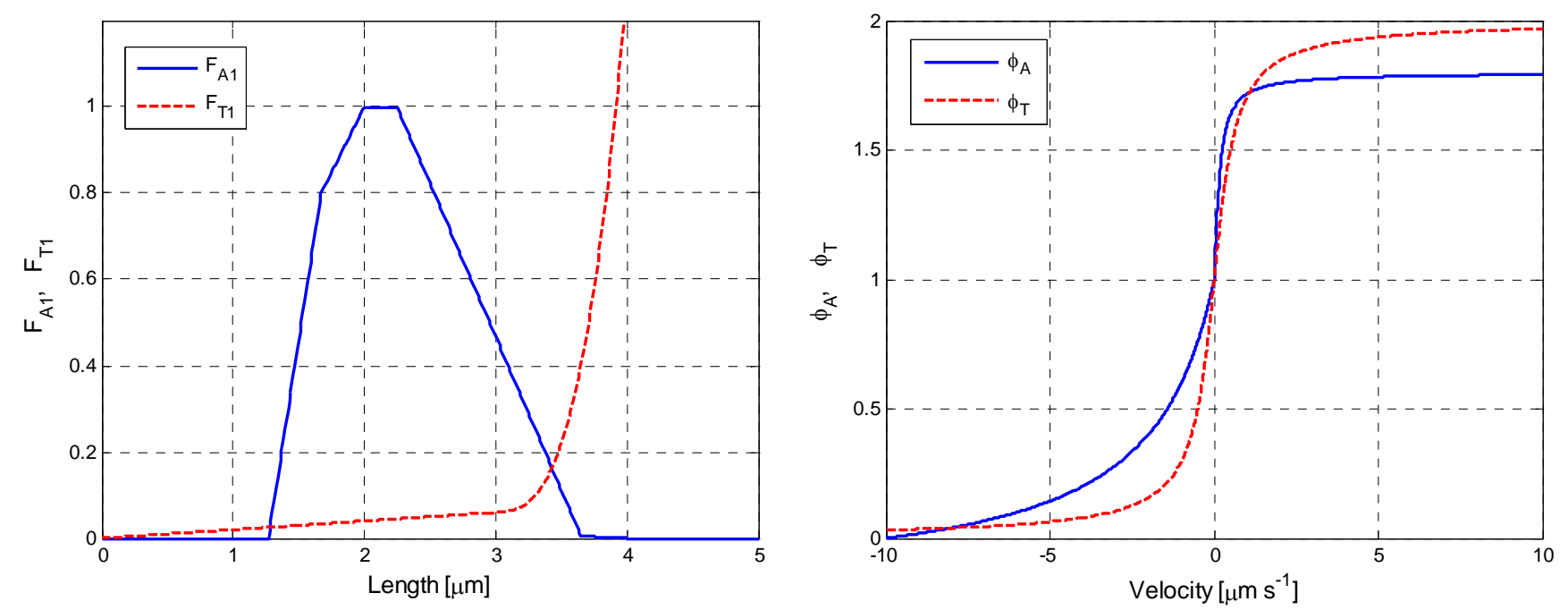

Fig. A.2: Force characteristics of the sarcomere active and passive elements

Typically the contribution of inertia forces (mass density $\sim 10^{3} \mathrm{~kg} \cdot \mathrm{m}^{-3}$ ) is a few orders of magnitude smaller than forces generated by the sarcomere, and can be safely neglected (Denoth, et al., 2002). Therefore, the governing equations for the mechanical response of a single sarcomere are: 


$$
\begin{aligned}
& F_{A}\left(L_{A}, \dot{L}_{A}\right)=F_{E}\left(L_{E}\right) \\
& F_{A}\left(L_{A}, \dot{L}_{A}\right)+F_{T}\left(L_{T}, \dot{L}_{T}\right)=F_{\text {ext }}(t)
\end{aligned}
$$

where $F_{\alpha}, L_{\alpha}(\alpha=A, E, T)$ are the forces and lengths associated with the active, elastic and passive components, respectively, and $\mathrm{F}_{\mathrm{ext}}$ is the contractile force generated by the sarcomere (or the external force applied on the sarcomere).

\section{A.2. Applicability of equation (4)}

Choosing the lengths of the active and passive elements as the state parameters, we use relations (A.1), (A.3), and (A.6) to write:

$$
\begin{array}{lll}
\phi_{A}\left(\dot{L}_{A}\right)=f_{1}\left(L_{A}, L_{T}\right) & ; & f_{1}\left(L_{A}, L_{T}\right) \equiv \frac{F_{E}\left(L_{T}-L_{A}\right)}{F_{A 1}\left(L_{A}\right)} \\
\phi_{T}\left(\dot{L}_{T}\right)=f_{2}\left(L_{A}, L_{T}, F_{\text {ext }}\right) & ; & f_{2}\left(L_{A}, L_{T}, F_{\text {ext }}\right) \equiv \frac{F_{\text {ext }}-F_{E}\left(L_{T}-L_{A}\right)}{F_{T 1}(L)} .
\end{array} .
$$

Since $\phi_{\mathrm{A}}$ and $\phi_{\mathrm{T}}$ are strictly monotonous, we can invert the above relations and write

$$
\dot{\mathbf{L}}=\mathbf{v}\left(\mathbf{L}, F_{\text {ext }}\right)
$$

consistent with (3). We note that here the relation described by (3) takes the form of a vector equation, and $\mathbf{L}$ is the vector of the state parameters.

\section{A.3. A simplified model}

We now introduce two simplifications to the single sarcomere model. We emphasize that these are not essential for our approach, but useful. Furthermore, we demonstrate the accuracy of the simplifications.

The first simplification treats the thick and thin filaments to be inextensible, as assumed by most theoretical studies and all mass-action kinetics models of the cross-bridge cycle. Recent experimental studies (Dunaway, et al., 2002; Goldman and Huxley, 1994; Wakabayashi, et al., 1994) show that the thin and thick filaments are compliant. However, the strain they suffer is very small compared to that generated by the sliding of the filaments during the muscle contraction. Indeed, numerical simulations (Denoth, et al., 2002) and Monte-Carlo simulations of muscle contraction (Chase, et al., 2004; Daniel, et al., 1998) that incorporate the observed filaments compliance show that the typical filaments strain is of the order of $0.2 \%$. Such strains 
may play an important role in the cross-bridge cycle kinetics, as suggested by Daniel et-al. (1998), by influencing the recruitment rate of cross-bridges due to myosin-actin binding site alignment. However, the significance of this effect with respect to the current work is of second order.

The second simplification is related to the force-velocity relation of the passive force element. The dominating passive force component in the sarcomere is the titin molecule. Experimental studies on single titin molecules (Kellermayer, et al., 1997; Rief, et al., 1997; Tskhovrebova, et al., 1997) demonstrate a nonlinear force-length relation attributed to a combination of entropic effects and extension of the polypeptide chain. However, the force-velocity relationship of the passive elements is not completely understood. Therefore, due to the significant uncertainty related to the force-velocity relation, combined with the similarity between $\mathrm{F}_{\mathrm{A} 2}$ and $\mathrm{F}_{\mathrm{T} 2}$ suggested by Denoth et al. (2002) and illustrated in Fig. A.2, it is reasonable to consider $\phi_{\mathrm{T}}=\phi_{\mathrm{A}}$.

Based on these assumptions, the response of the sarcomere is governed by

$$
F_{\text {ext }} \cong\left(F_{A 1}(L)+F_{T 1}(L)\right) \cdot \phi(\dot{L})=F_{1}(L) \cdot \phi(\dot{L})
$$

where $\mathrm{L}$ is the sarcomere length and $\phi=\phi_{\mathrm{A}}$.

Fig. A.3 shows a comparison of the simplified model (A.9) with the original model of Denoth et al. (2002) under a sinusoidal applied force $F_{\text {ext }}=1+\frac{1}{2} \sin (5 \pi t)$. As expected, the simplified model is a good approximation for the original model. In particular the contribution of the elastic element is completely negligible. Furthermore, the fully simplified model is acceptable in light of the experimental uncertainties with respect to $\phi_{\mathrm{T}}$ and $\phi_{\mathrm{A}}$. 

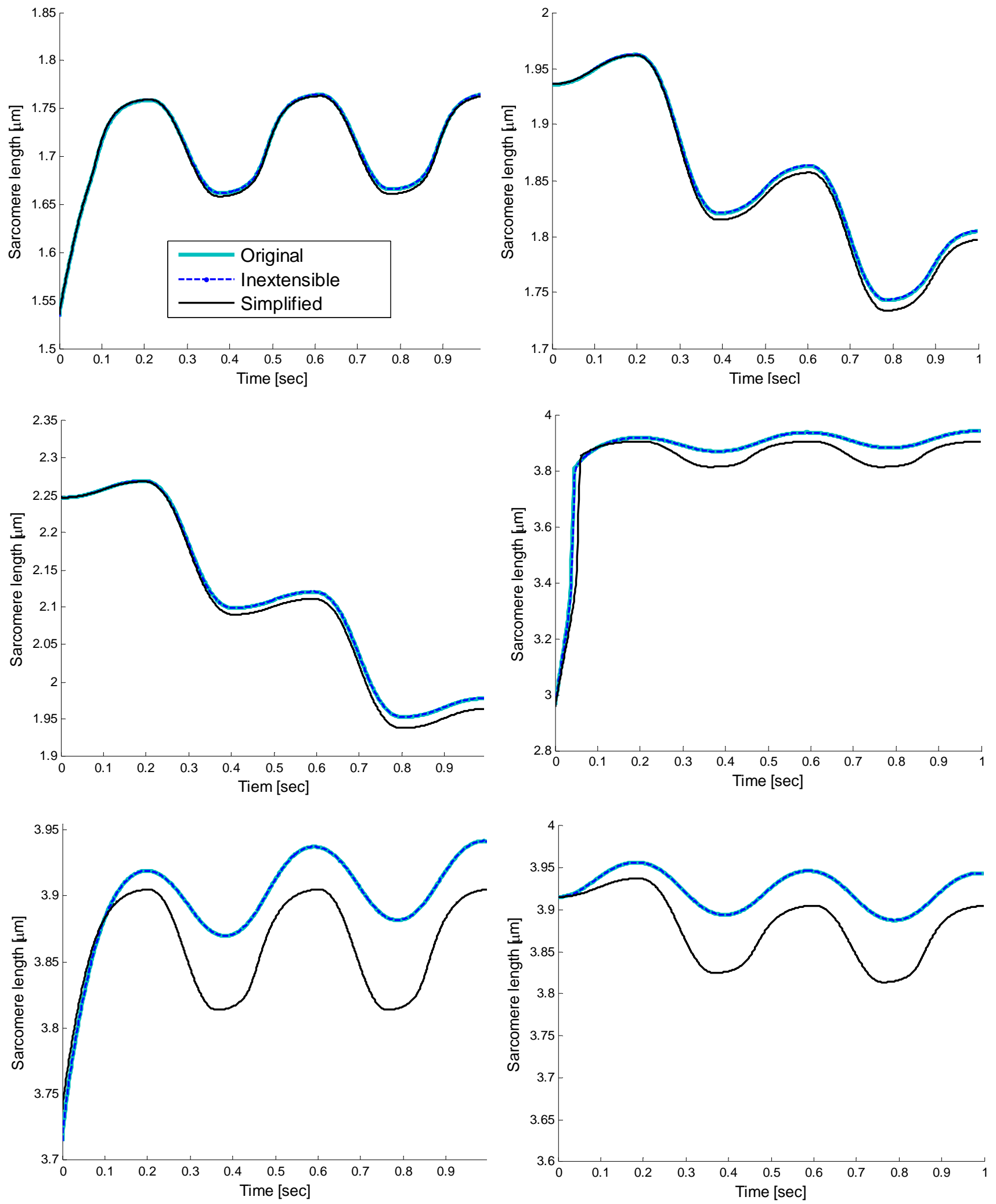

Fig. A.3: A comparison of the three sarcomere models under sinusoidal force control starting from various initial lengths. 


\section{$\underline{\text { References }}$}

Allinger, T.L., Epstein, M., Herzog, W., 1996. Stability of muscle fibers on the descending limb of the force-length relation. A theoretical consideration. Journal of Biomechanics 29 (5), 627-633.

Brown, L.M., Hill, L., 1982. Mercuric-Chloride in Alcohol and Chloroform Used as a Rapidly Acting Fixative for Contracting Muscle-Fibers. Journal of Microscopy-Oxford 125 (MAR), 319-336.

Caughey, T.K., 1963. Derivation and Application of Fokker-Planck Equation to Discrete Nonlinear Dynamic Systems Subjected to White Random Excitation. Journal of the Acoustical Society of America 35 (11), 1683-\&.

Chase, P.B., Macpherson, J.M., Daniel, T.L., 2004. A spatially explicit nanomechanical model of the half-sarcomere: Myofilament compliance affects Ca2+-Activation. Annals of Biomedical Engineering 32 (11), 1559-1568.

Daniel, T.L., Trimble, A.C., Chase, P.B., 1998. Compliant realignment of binding sites in muscle: Transient behavior and mechanical tuning. Biophysical Journal 74 (4), 1611-1621.

Denoth, J., Stussi, E., Csucs, G., Danuser, G., 2002. Single muscle fiber contraction is dictated by inter-sarcomere dynamics. Journal of Theoretical Biology 216 (1), 101-122.

Dobbie, I., Linari, M., Piazzesi, G., Reconditi, M., Koubassova, N., Ferenczi, M.A., Lombardi, V., Irving, M., 1998. Elastic bending and active tilting of myosin heads during muscle contraction. Nature 396 (6709), 383-387.

Dunaway, D., Fauver, M., Pollack, G., 2002. Direct measurement of single synthetic vertebrate thick filament elasticity using nanofabricated cantilevers. Biophysical Journal 82 (6), 3128-3133.

Edman, K.A.P., Reggiani, C., 1984. Redistribution of Sarcomere-Length During Isometric Contraction of Frog-Muscle Fibers and Its Relation to Tension Creep. Journal of PhysiologyLondon 351 (JUN), 169-198.

Goldman, Y.E., Huxley, A.F., 1994. Actin Compliance - Are You Pulling My Chain. Biophysical Journal 67 (6), 2131-2133.

Gordon, A.M., Huxley, A.F., Julian, F.J., 1966a. Tension Development in Highly Stretched Vertebrate Muscle Fibres. Journal of Physiology-London 184 (1), 143-\&.

Gordon, A.M., Huxley, A.F., Julian, F.J., 1966b. Variation in Isometric Tension with Sarcomere Length in Vertebrate Muscle Fibres. Journal of Physiology-London 184 (1), 170-+.

Harry, J.D., Ward, A.W., Heglund, N.C., Morgan, D.L., McMahon, T.A., 1990. CrossBridge Cycling Theories Cannot Explain High-Speed Lengthening Behavior in Frog-Muscle. Biophysical Journal 57 (2), 201-208.

Hill, A.V., 1938. The heat of shortening and the dynamic constants of muscle. Proceedings of the Royal Society of London Series B-Biological Sciences 126 (843), 136-195.

Huxley, A.F., 1957. Muscle Structure and Theories of Contraction. Progress in Biophysics \& Molecular Biology 7, 255-\&. 
Huxley, A.F., Niedergerke, R., 1954. Structural Changes in Muscle During Contraction Interference Microscopy of Living Muscle Fibres. Nature 173 (4412), 971-973.

Huxley, H., Hanson, J., 1954. Changes in the Cross-Striations of Muscle During Contraction and Stretch and Their Structural Interpretation. Nature 173 (4412), 973-976.

Irving, M., Lombardi, V., Piazzesi, G., Ferenczi, M.A., 1992. Myosin Head Movements Are Synchronous with the Elementary Force-Generating Process in Muscle. Nature 357 (6374), 156158.

Irving, M., Piazzesi, G., Lucii, L., Sun, Y.B., Harford, J.J., Dobbie, I.M., Ferenczi, M.A., Reconditi, M., Lombardi, V., 2000. Conformation of the myosin motor during force generation in skeletal muscle. Nature Structural Biology 7 (6), 482-485.

Iwazumi, T., 1987. High-Speed Ultrasensitive Instrumentation for Myofibril Mechanics Measurements. American Journal of Physiology 252 (2), C253-C262.

Julian, F.J., Morgan, D.L., 1979a. Effect on Tension of Nonuniform Distribution of Length Changes Applied to Frog-Muscle Fibers. Journal of Physiology-London 293 (AUG), 379-\&.

Julian, F.J., Morgan, D.L., 1979b. Intersarcomere Dynamics During Fixed-End Tetanic Contractions of Frog-Muscle Fibers. Journal of Physiology-London 293 (AUG), 365-378.

Katz, B., 1939. The relation between force and speed in muscular contraction. Journal of Physiology-London 96 (1), 45-64.

Kellermayer, M.S.Z., Smith, S.B., Granzier, H.L., Bustamante, C., 1997. Folding-unfolding transitions in single titin molecules characterized with laser tweezers. Science 276 (5315), $1112-$ 1116.

LeVeque, R.J., 1998. Numerical Methods for Conservation Laws. Birkhauser Verlag, Basel, Switzerland.

LeVeque, R.J., 2002. Finite Volume Methods for Hyperbolic Problems Cambrige University Press, Cambridge, UK.

Lombardi, V., Piazzesi, G., 1990. The Contractile Response During Steady Lengthening of Stimulated Frog-Muscle Fibers. Journal of Physiology-London 431, 141-171.

Lombardi, V., Piazzesi, G., 1992. Force Response in Steady Lengthening of Active Single Muscle Fibers. In: Simmons, R.M. (Ed.), Muscular Contraction. Cambridge university press, Cambridge.

Marland, E., 1998. Dynamics of the sarcomere. Univ. of Utah, Salt Lake City.

Morgan, D.L., 1990. New insights into the behavior of muscle during active lengthening. pp. 209-221.

Morgan, D.L., Allen, D.G., 1999. Early events in stretch-induced muscle damage. Journal of Applied Physiology 87 (6), 2007-2015.

Morgan, D.L., Mochon, S., Julian, F.J., 1982. A Quantitative Model of Inter-Sarcomere Dynamics During Fixed-End Contractions of Single Frog-Muscle Fibers. Biophysical Journal 39 (2), 189-196. 
Morgan, D.L., Proske, U., 1984. Mechanical-Properties of Toad Slow Muscle Attributed to Non-Uniform Sarcomere Lengths. Journal of Physiology-London 349 (APR), 107-117.

Morgan, D.L., Proske, U., 2004. Popping sarcomere hypothesis explains stretch-induced muscle damage. Clinical and Experimental Pharmacology and Physiology 31 (8), 541-545.

Mutungi, G., Ranatunga, K.W., 2000. Sarcomere length changes during end-held (isometric) contractions in intact mammalian (rat) fast and slow muscle fibres. Journal of Muscle Research and Cell Motility 21 (6), 565-575.

Oksendal, B.K., 2003. Stochastic Differential Equations: An Introduction with Applications. Springer, Berlin.

Pate, E., Cooke, R., 1989. A Model of Crossbridge Action - the Effects of Atp, Adp and Pi. Journal of Muscle Research and Cell Motility 10 (3), 181-196.

Piazzesi, G., Francini, F., Linari, M., Lombardi, V., 1992. Tension Transients During Steady Lengthening of Tetanized Muscle-Fibers of the Frog. Journal of Physiology-London 445, 659711.

Piazzesi, G., Reconditi, M., Linari, M., Lucii, L., Sun, Y.B., Narayanan, T., Boesecke, P., Lombardi, V., Irving, M., 2002. Mechanism of force generation by myosin heads in skeletal muscle. Nature 415 (6872), 659-662.

Rief, M., Gautel, M., Oesterhelt, F., Fernandez, J.M., Gaub, H.E., 1997. Reversible unfolding of individual titin immunoglobulin domains by AFM. Science 276 (5315), 1109-1112.

Risken, H., 1989. The Fokker-Planck Equation. Springer, Berlin.

Talbot, J.A., Morgan, D.L., 1996. Quantitative analysis of sarcomere non-uniformities in active muscle following a stretch. Journal of Muscle Research and Cell Motility 17 (2), 261-268.

Telley, I.A., Denoth, J., 2007. Sarcomere dynamics during muscular contraction and their implications to muscle function. Journal of Muscle Research and Cell Motility 28 (1), 89-104.

Telley, I.A., Stehle, R., Ranatunga, K.W., Pfitzer, G., Stussi, E., Denoth, J., 2006. Dynamic behaviour of half-sarcomeres during and after stretch in activated rabbit psoas myofibrils: sarcomere asymmetry but no 'sarcomere popping'. Journal of Physiology-London 573 (1), 173185.

Thorson, J., White, D.C.S., 1969. Distributed Representations for Actin-Myosin Interaction in Oscillatory Contraction of Muscle. Biophysical Journal 9 (3), 360-\&.

Tskhovrebova, L., Trinick, J., Sleep, J.A., Simmons, R.M., 1997. Elasticity and unfolding of single molecules of the giant muscle protein titin. Nature 387 (6630), 308-312.

Wakabayashi, K., Sugimoto, Y., Tanaka, H., Ueno, Y., Takezawa, Y., Amemiya, Y., 1994. X-Ray-Diffraction Evidence for the Extensibility of Actin and Myosin-Filaments During MuscleContraction. Biophysical Journal 67 (6), 2422-2435.

White, D.C.S., Thorson, J., 1973. The Kinetics of Muscle Contraction. Progress in Biophysics \& Molecular Biology 27, 173-255.

Wray, J.S., 1979. Structure of Backbone in Myosin-Filaments of Muscle. Nature 277 (5691), $37-40$. 
Zahalak, G.I., 1997. Can muscle fibers be stable on the descending limbs of their sarcomere length-tension relations? Journal of Biomechanics 30 (11-12), 1179-1182. 


\section{Figure captions}

Fig. 1: Schematic illustration of a single sarcomere. Contraction occurs due to relative sliding between the thin (actin) and thick (myosin) filaments, and force is generated by myosin heads that bind to the thin filaments

and form cross-bridges.

Fig. 2: Force-length relation of a single sarcomere under isometric conditions................................................... 6

Fig. 3: The force-velocity relation of a single sarcomere at ideal length. The force is normalized by the isometric force. Negative velocities correspond to shortening............................................................................... 7

Fig. 4: Velocity as a function of sarcomere length at a fixed force. For the smaller force, there are two stable sarcomere lengths $\left(v(L)=0, v^{\prime}(L) \leq 0\right)$ at 1.63 and $3.83 \mu \mathrm{m}$. There is only one stable sarcomere length $(\mathrm{L}=4.08 \mu \mathrm{m})$ for the higher force.

Fig. 5: Steady state solution of the Fokker-Planck equation (16) with $D=10^{-3} \mu \mathrm{m}^{2} / \mathrm{sec}$ and $\mathrm{F}_{\text {ext }}=1$. Comparison between analytical solution (19), and length distribution after 20 seconds of simulation using a high order finite volume numerical scheme.

Fig. 6: Isometric force $\widetilde{F}_{1}$ for two sarcomeres with a different value of force capacity factor $\mathrm{C}$. The isometric force generated by the weaker sarcomere, $\mathrm{C}^{(1)}$, is always smaller for the same length....................................... 15

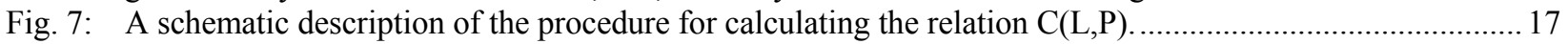

Fig. 8: Finite volume method: a schematic illustration of the cells and associated "reverse" characteristics at their boundaries. Arrows are used to illustrate probability flow through the cell boundary.

Fig. 9: Simulation of an eccentric contraction protocol studied by Talbot and Morgan (1996). (a) The resultant force. Inset: Imposed length vs. time. (b) Final distribution of sarcomere lengths and comparison between experimental observation (histogram, based on results of Talbot and Morgan (1996)) and the results of simulation. (c,d) Evolution in time of the length distribution predicted by Models $\mathrm{F}$ and $\mathrm{V}$ respectively. ...22

Fig. 10: Simulation of the eccentric contraction protocol on the ascending limb studied by Talbot and Morgan (1996). (a) The resultant force. Inset: Imposed length vs. time. (b) Comparison between experimental observations (histogram, based on results of Talbot and Morgan (1996)) and the results of simulation....... 24

Fig. 11: Sarcomere popping due to an increase of the external force to $1.7 \mathrm{~F}_{0}$. (a) Accumulation of overstretched sarcomeres. inset: external force exerted on the myofibril. (b) Evolution of the length distribution for Model V. 25

Fig. 12: Force controlled stretch-release experiment followed by force recovery after 5 seconds: (a) Initial distribution. Inset: external force vs. time. (b) Fraction of overstretched sarcomeres (c) Average sarcomere length (proportional to the overall myofibril length) (d) Evolution in time of the length distribution associated with Model V.....

Fig. 13: Force controlled stretch-release experiment followed by force recovery after 2 seconds: (a) External force vs. time. (b) Fraction of overstretched sarcomeres (c) Average sarcomere length (proportional to overall myofibril length) (d) Evolution in time of the length distribution associated with Model V........................ 28

Fig. 14: Myofibril response to periodic length control. (a) Accumulation of overstretched sarcomeres. (b) Force response after 20 cycles. Inset: Schematic illustration of the imposed cyclic length.

Fig. 15: Fraction of overstretched sarcomeres for a myofibril subjected to a cyclic force. (a) Accumulation of overstretched sarcomeres vs. time for different load frequencies with $\mathrm{f}_{\min }=1, \mathrm{f}_{\max }=1.45$. Inset: Accumulation of overstretched sarcomeres vs. number of cycles. (b) Accumulation of overstretched sarcomeres in the first 10 seconds for $\mathrm{f}_{\min }=1$ and $\mathrm{f}_{\max }=1.45$. Inset: Schematic illustration of the imposed cyclic force. (c) Accumulation of overstretched sarcomeres for different values of $f_{\max }$ and $f_{\min }=1, T=1$ second. (d) The fraction of overstretched sarcomeres after 10,000 cycles as a function of $\mathrm{f}_{\min }$. 